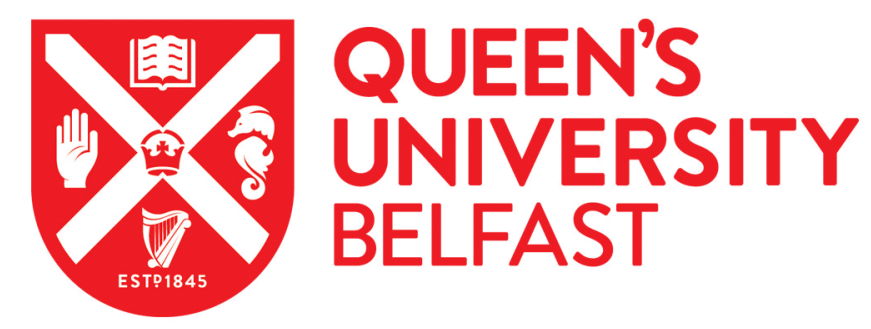

\title{
Long Span Bridges: Enhanced data fusion of GPS displacement and deck accelerations
}

Xu, Y., brownjohn, J., Hester, D., \& Koo, K. (2017). Long Span Bridges: Enhanced data fusion of GPS displacement and deck accelerations. Engineering Structures, 147.

\section{Published in:}

Engineering Structures

\section{Document Version:}

Peer reviewed version

\section{Queen's University Belfast - Research Portal:}

Link to publication record in Queen's University Belfast Research Portal

\section{Publisher rights}

Copyright 2017 Elsevier.

This manuscript is distributed under a Creative Commons Attribution-NonCommercial-NoDerivs License

(https://creativecommons.org/licenses/by-nc-nd/4.0/), which permits distribution and reproduction for non-commercial purposes, provided the author and source are cited.

\section{General rights}

Copyright for the publications made accessible via the Queen's University Belfast Research Portal is retained by the author(s) and / or other copyright owners and it is a condition of accessing these publications that users recognise and abide by the legal requirements associated with these rights.

Take down policy

The Research Portal is Queen's institutional repository that provides access to Queen's research output. Every effort has been made to ensure that content in the Research Portal does not infringe any person's rights, or applicable UK laws. If you discover content in the Research Portal that you believe breaches copyright or violates any law, please contact openaccess@qub.ac.uk. 


\title{
Long-Span Bridges: Enhanced data fusion of GPS displacement and
}

\section{deck accelerations}

\author{
Yan Xu ${ }^{\mathrm{a}}$, James Brownjohn ${ }^{\mathrm{a}}$, David Hester ${ }^{\mathrm{b}}, \mathrm{Ki} \mathrm{Koo}^{\mathrm{a}}$ \\ ${ }^{a}$ Vibration Engineering Section, College of Engineering, Mathematics and Physical Sciences, \\ University of Exeter, UK \\ ${ }^{b}$ Department of Civil Engineering, Queen's University Belfast, UK
}

\begin{abstract}
Displacement data under operational loads are an important aid for the estimation of structural performance, but accurate measurement of structural displacement remains as a challenging task, especially for long-span bridges. The global positioning system (GPS) is the common choice for longspan bridge displacement monitoring but the measurement accuracy is not satisfactory. The purpose of this study is to improve the GPS accuracy using a practical data fusion method. Although the main algorithms of data fusion method based on multi-rate Kalman filter are already reported, the detail about how to select the noise parameters required for Kalman filter is not provided. This paper demonstrates that maximum likelihood estimation (MLE) can be used to determine the necessary noise parameters. The proposed approach was validated on numerical and field data, the latter from a single-day displacement monitoring campaign on the Humber Bridge in the UK. The direct measurement by GPS was merged with the collocated acceleration data and the resulting displacement signal was evaluated by comparing it to the displacement signal from an independent vision-based system. Through the comparison, it is shown that MLE enhanced data fusion is practical to improve the GPS measurement accuracy and to widen the frequency bandwidth. The MLE provides an estimation about the GPS noise (assumed as zero-mean Gaussian process) with the standard deviation varying from $6 \mathrm{~mm}$ to $16 \mathrm{~mm}$ in the test day. The normalised root mean square deviation of GPS
\end{abstract}


measurement compared with the vision-based measurement was decreased from $3.17 \%$ to $2.37 \%$ after applying the data fusion.

Keywords: long-span bridge; GPS; vision-based system; Kalman filter; maximum likelihood estimation.

\section{Introduction}

Long-span bridges are vital links in the transportation networks. Structural degradation and increasing service demands such as increased traffic and heavier vehicle loads make it important to monitor the status of in-service bridges. The occurrence of any bridge failure could cause disruption of traffic network and even more serious consequences (e.g. loss of human lives and huge economic cost). However, the inspection process to determine bridge performance and prioritise maintenance is necessary but very costly.

Bridge deformation information is a significant metric for bridge performance assessment [1]. For example, measurement of deformation during controlled vehicle load testing helps to estimate bridge load carrying capacity [2] [3]. Serviceability is reflected through deformation during normal operation, since extreme values and ranges indicate problems that may limit operational use.

\subsection{Current sensing technologies for bridge deformation monitoring}

Measuring the deformation in long-span bridges is a significant but challenging task. The conventional sensors like linear variable differential transformers (LVDT) are infeasible due to requirement of direct access to bridges and the availability of a stationary reference point. Doubleintegration of acceleration data works well only for short-time signals within several seconds or a few minutes depending on the signal to noise ratio. For longer bridges requiring longer time scales for deformation, integration schemes typically fail to identify the static and quasi-static component of displacement. Robotic Total Stations (RTS) have been validated to be feasible for monitoring the semi-static and dynamic displacement of relatively stiff structure $[4,5]$. Optical systems using computer vision approaches have the potential to identify the bridge deformation at high sample rate and good accuracy level $[6,7]$. However, for any optical based systems, the application for long-term 
monitoring (e.g. several days, months) is limited due to the requirement of unobstructed view as well as the measurement accuracy dependent on atmospheric refraction and turbulence [8].

Currently the global positioning system (GPS) is basis for the common sensing of displacement in long-span bridges [1]. However, the accuracy level of GPS data is a major concern. Nickitopoulou, Protopsalti and Stiros [9] suggest standard accuracy of up to $15 \mathrm{~mm}$ and $35 \mathrm{~mm}$ for horizontal and vertical measurements, respectively, at 98.5 percentile level without gross errors such as cycle slip or multipath. Casciati and Fuggini [10] confirmed the accuracy to be of the order of sub-centimetre level through both static and dynamic tests. Recently high-rate GPS receivers e.g. up to $100 \mathrm{~Hz}$ are available due to the developments in GNSS receiver and antenna technologies [11], but the increase of sample rate may not provide any improvement, and may even degrade accuracy [12]. These tests were conducted in an open area with no reflective surface whereas for the field implementations especially in long-span cable-stayed or suspension bridges, the measurement accuracy becomes worse since the multipath effects near cables and deck are more obvious [9].

\subsection{Previous efforts to improve GPS accuracy}

An effective way to improve the GPS measurement accuracy is data fusion which allows the integration of data from a series of sensors to make a better estimation than that from any sensor alone [13]. Efforts have been made to integrate the GPS measurement with other sensors (commonly accelerometers). Li et al. [14] demonstrated the complementary feature of GPS and accelerometer measurement in low-frequency and high-frequency ranges through analysing the in-site measurement of a tall building during the earthquake and typhoon. Roberts et al. [15] proposed a sensor configuration to ensure the time synchronisation and measurement axis alignment between two types of sensors. The post processing methods for data fusion include: 1) integrating acceleration signals to displacement while correcting the result using GPS-derived initial condition [15], 2) reconstructing displacement using the low-frequency component derived from GPS measurement and highfrequency component derived from acceleration measurement [16], and 3) denoising GPS measurement (filter) based on the dominant frequencies derived from acceleration signals [10][19][20]. Another effort is linking the GPS measurement with Robotic Total Station (RTS) 
measurement through superimposition of semi-static displacement from RTS and dynamic displacement from GPS [17] or a supervised learning approach to distinguish the oscillation amplitude from the measurement noise [21]. These approaches are supervised learning process with filter or correction and thus require the users' judgement and involvement for the selection of certain 'parameters', e.g. determining the cut-off frequency for signal denoising or selecting the effective frequency bandwidth of measured acceleration and displacement signals for time-history superimposition.

A promising approach to link autonomously GPS measurement with acceleration signals is to use Kalman filter, an approach that has been actively applied in the fields of navigation, computer vision and economics, etc. In the structural monitoring field, Smyth and $\mathrm{Wu}$ [22] proposed a multi-rate Kalman filter approach to fuse the measured acceleration and displacement signals at different sample rates while Chang and Xiao [23] applied the method to a pedestrian bridge by integrating the data from the accelerometer and a vision-based system. Kim et al. [24] modified this fusion method by adding acceleration measurement bias into state variables. In the Kalman filter based fusion methods, there is no displacement base correction nor any frequency-selective filter for noise reduction. Instead, the Kalman filter method finds the best estimate at each time step by weighted average between the noisy displacement measurement and the recursive prediction made from previous best estimate. This estimation process requires reasonably accurate models of the noise in both displacement measurement and state transition model (e.g. measurement and process noise covariances). However, these noise covariances are assumed as known parameters in the abovementioned works, which in many situations is not the case. Therefore, Li and Chang [25] proposed an adaptive subspace technique to quantify time-sensitive variances of noise embedded in measurement signals whereas this requires knowledge of the structure dynamic characteristics and multi-point measurement data, which again is not always available.

\subsection{Focus of this study}

From the above text it is evident that challenges remain on how to measure the displacement in longspan bridges using GPS with suitable measurement accuracy and acquisition frequency. It has been 
shown previously that a Kalman filter can be used to merge displacement data with corresponding acceleration data to improve displacement measurement accuracy [22] provided that noise parameters such as process and measurement noise covariances are known. Therefore this study proposes the novel step of using maximum likelihood estimation (MLE) to accurately estimate the noise parameters required by the Kalman filter process.

The effectiveness of the proposed approach is first demonstrated on numerically simulated data of a single degree of freedom system. Afterwards, this method is applied to improve the accuracy of GPS measurement recorded on the Humber Bridge, UK. To examine the effectiveness of the approach when applied to field data, the results are compared to the displacement recorded using an independent vision-based system. Although the vision-based system has some limitations e.g. requirement of good environment and clear view, the system performance on a calm day with stable observation conditions was validated to be satisfactory over several hours in day time through midspan displacement monitoring tests in long-span bridges including the Vincent Thomas Bridge, USA [26], Tsing Ma Bridge, China [27] and P25A bridge, Lisbon [28].

In the past other researchers have looked at the possibility of ameliorating GPS displacement data by fusing it with corresponding acceleration data, so the main purpose of this paper is to investigate the improvement in the accuracy of displacement readings that can be achieved by incorporating MLE in data fusion process.

To this end, Section 2 gives an overview of the data fusion method based on the Kalman filter algorithm and the principles of maximum likelihood estimation while Section 4 describes the field testing at Humber Bridge, UK and the results observed.

\section{Data fusion of acceleration and displacement}

In effect this paper proposes to combine the work of Smyth and $\mathrm{Wu}$ [22] with maximum likelihood estimation (MLE), and a brief overview of each is given below. 
Smyth and $\mathrm{Wu}[22]$ utilise the multi-rate Kalman filter to fuse the acceleration and displacement measurement with different sample rate for an improved estimation of displacement signals. (Specific details on their approach are provided in Section 2.1). In this method, users need to specify the noise parameters e.g. process and measurement noise covariances ( $\mathrm{Q}$ and $\mathrm{R}$ respectively in this paper). These parameters are related to the measurement noise in measured displacement and acceleration signals. The difficulty is that these noise parameters are in fact unknown and the erroneous selection of noise parameters leads to poor performance of displacement estimates.

MLE is a common method in statistics to estimate the parameters of a statistical model (e.g. mean and standard deviation) from an incomplete sample of observed data i.e. data on the whole population is not available. In this paper, MLE is used to estimate the parameters related to process and measurement noise covariances required by the method of Smyth and Wu [22]. Further information on MLE is presented in Section 2.2 below.

\subsection{Multi-rate Kalman filter with backward smoothing}

Smyth and $\mathrm{Wu}[22]$ developed a method to merge measured displacement data with collocated acceleration data to obtain improved estimation of displacement. The method uses the multi-rate Kalman filter with forward estimation and backward smoothing. Summary details are reproduced here to highlight issues concerning some noise parameters that are required but unknown, and to define a number of variables discussed subsequently.

State variables for the discrete-time state space model are introduced as displacement and velocity:

$$
\mathbf{X}(k)=[\mathrm{x}(k), \dot{\mathrm{x}}(k)]^{\mathrm{T}} .
$$

The dynamic motion can be predicted recursively in a linear state-space representation as,

$$
\mathbf{X}(k+1)=\mathrm{A} \mathbf{X}(k)+\mathrm{B} u(k)+w(k)
$$

and the displacement measurement is modelled as a Gaussian process,

$$
z(k)=\mathrm{H} \mathbf{X}(k)+v(k)
$$


where $k$ denotes the time step; and $u(k)$ and $z(k)$ are the measured data of acceleration and displacement, respectively. The matrices $\mathrm{A}, \mathrm{B}$ and $\mathrm{H}$ are given by

$$
\mathrm{A}=\left[\begin{array}{cc}
1 & d t \\
0 & 1
\end{array}\right], \mathrm{B}=\left[\begin{array}{c}
d t^{2} / 2 \\
d t
\end{array}\right], \mathrm{H}=\left[\begin{array}{ll}
1 & 0
\end{array}\right]
$$

where $d t$ is the sampling time interval of acceleration signals.

The parameter $w(k)$ is the uncertainty in state-transition model or the process noise assumed as a two-dimensional Gaussian process with zero mean and fixed covariance Q. The parameter $v(k)$ denotes the measurement noise assumed as a white noise Gaussian process with zero mean and fixed variance $\mathrm{R}$. The covariance matrices $\mathrm{Q}$ and $\mathrm{R}$ can be obtained by

$$
\mathrm{Q}=\left[\begin{array}{cc}
q d t^{3} / 3 & q d t^{2} / 2 \\
q d t^{2} / 2 & q d t
\end{array}\right], \mathrm{R}=\frac{r}{d t}
$$

where $q$ and $r$ denote the variance of acceleration and displacement noise in the analogous continuous-time system. It is noted that the GPS observations are dominated by both the white and the coloured noise (due to systematic errors) [29], but here only the white noise is modelled since the systematic errors during a short duration possibly have nearly constant amplitudes [21].

The discrete-time Kalman filter algorithm and backward smoothing technique can be applied for the state estimation (the mean $\hat{\mathbf{X}}$ and the covariance matrix $\mathbf{P}$ of optimal estimate of state variables) including three main steps, time update, measurement update and backward smoothing. The corresponding details could refer to [22].

During the implementation of Kalman filter, parameters modelling the uncertainty of state-transition model and measurement model (e.g. Q and $\mathrm{R}$ or $q$ and $r$ ) need to be determined ahead before any state estimation. Obviously, the accuracy level of the state estimation depends on the selection of these parameters, but unfortunately the true value of these variables is typically unknown in practice.

In our state model, the parameters $q$ and $r$ are the noise variances of acceleration and displacement in the continuous-time model. Ideally the noise variances could be estimated by taking some stationary measurement and analysing the acquired data, but the stationary measurement is not always available, 
especially in a field test. Also the noise level might not be constant with time and space, e.g. the GPS noise level becomes larger in the case of narrower distribution of satellites at certain times of the day.

It is essential to find an appropriate way to determine the noise parameters needed for Kalman filter. In previous work on this topic either the procedure for determining the noise parameters is not given $[22,24]$ or they are estimated by the energy of noise component separated from the noisy signals using an adaptive subspace technique [25]. This subspace method requires the input of multi-channel measurement in which the number of channels is at least equal to the number of identifiable modes, which is neither particularly satisfactory nor practical. Therefore in the next section, the MLE method is presented and is used to tune the noise parameters and initial state of state variable before application of the Kalman filter.

\subsection{Maximum likelihood estimation (MLE) of noise parameters}

The MLE is a common method to estimate the unknown parameters of a statistical model (e.g. mean and standard deviation) when some observational data are available. In essence, the approach works by

(i) assuming a particular statistical distribution model (e.g. normal distribution) for an event with some unknown statistical parameters,

(ii) collecting observational data about the event, and

(iii) carrying out a parametric study to identify the model parameters (e.g. mean and standard deviation) so that the possibility of getting observed data under this parameter choice is greater than that under any other choice.

Thus the MLE is an optimisation process to find the value of unknown parameters in a statistic model that maximises the likelihood of this statistic model given the observational data. The following equations and text summarise the main mathematical steps in the process. The statistic event here is corresponding to the displacement information at each time step. The statistical model and inherent unknown parameters are provided in Equation(6) and Equation(7). Equations (8)-(9) give the 
calculation formulas for log-likelihood function that will be maximised to derive the optimal estimate of unknown model parameters.

In our application, the observational data $\left\{\mathbf{Z}_{\mathrm{N}}\right\}$ are a sequence of displacement measurements $\mathrm{z}_{k}(k=1,2, \ldots, \mathrm{N})$. The displacement measurement is a Gaussian process with the probability density derived from the measurement equation(3) in the Kalman filter referred to as [30],

$$
\begin{aligned}
& p\left(\mathrm{z}_{k} \mid \boldsymbol{\theta}\right)=(2 \pi)^{-1} \operatorname{det}\left(\mathrm{HP}(k \mid k-1) \mathrm{H}^{\mathrm{T}}+\mathrm{R}\right)^{-1 / 2} \times \\
& \quad \exp \left(-\frac{1}{2}[z(k)-\mathrm{H} \hat{\mathbf{X}}(k \mid k-1)]^{\mathrm{T}}\left(\mathrm{HP}(k \mid k-1) \mathrm{H}^{\mathrm{T}}+\mathrm{R}\right)^{-1}[z(k)-\mathrm{H} \hat{\mathbf{X}}(k \mid k-1)]\right)
\end{aligned}
$$

The symbol $\boldsymbol{\theta}$ represents the unknown parameters in the statistical model of displacement measurement including i) the noise variances $q$ and $r$ of measured acceleration and displacement in the continuous-time system, and ii) the initial state of state variables $\mathbf{X}(0)$. Thus,

$$
\boldsymbol{\theta}=[q, r, \mathbf{X}(0)] .
$$

The likelihood of the parameters in a statistical model given the observational data is equal to the probability of those observed data given the model parameters shown in the following equation,

$$
l\left(\boldsymbol{\theta} \mid \mathbf{Z}_{\mathbf{N}}\right)=p\left(\mathbf{Z}_{\mathbf{N}} \mid \boldsymbol{\theta}\right)=\prod_{k=1}^{N} p\left(\mathrm{z}_{k} \mid \boldsymbol{\theta}\right)
$$

Since the natural logarithm of likelihood function or log-likelihood is monotonically related to the likelihood function, the log-likelihood function is deduced using Equations (6) and (8) for computational convenience,

$$
\begin{aligned}
\ln l\left(\boldsymbol{\theta} \mid \mathbf{Z}_{\mathrm{N}}\right) & =-N \ln (2 \pi)-\frac{1}{2} \sum_{k=1}^{N} \ln \operatorname{det}\left(\mathrm{H} \mathbf{P}(k \mid k-1) \mathrm{H}^{\mathrm{T}}+\mathrm{R}\right) \\
& -\frac{1}{2} \sum_{k=1}^{N}[z(k)-\mathrm{H} \hat{\mathbf{X}}(k \mid k-1)]^{\mathrm{T}}\left(\mathrm{H} \mathbf{P}(k \mid k-1) \mathrm{H}^{\mathrm{T}}+\mathrm{R}\right)^{-1}[z(k)-\mathrm{H} \hat{\mathbf{X}}(k \mid k-1)]
\end{aligned}
$$

The optimal estimate of unknown parameters $\boldsymbol{\theta}^{*}$ is derived by maximising the log-likelihood function using nonlinear optimisation algorithm,

$$
\boldsymbol{\theta}^{*}=\operatorname{argmax} \ln l\left(\boldsymbol{\theta} \mid \mathbf{Z}_{\mathbf{N}}\right)
$$


The log-likelihood function can have many local maxima. In order to get a global maximum, a large sets of initial model parameters $\boldsymbol{\Theta}^{0}=\left\{\boldsymbol{\theta}_{1}^{0}, \boldsymbol{\theta}_{2}^{0}, \ldots, \boldsymbol{\theta}_{\mathrm{M}}^{0}\right\}$ are generated based on the uniform distribution within a specified lower and upper bounds. For each set of initial vector $\boldsymbol{\theta}_{\mathrm{j}}^{0}$, an optimum $\boldsymbol{\theta}_{\mathrm{j}}^{*}$ is found using the MLE method. By comparing the log-likelihood of all derived optima $\boldsymbol{\Theta}^{*}=\left\{\boldsymbol{\theta}_{1}^{*}, \boldsymbol{\theta}_{2}^{*}, \ldots, \boldsymbol{\theta}_{\mathrm{M}}^{*}\right\}$, the one corresponding to the maximum log-likelihood is taken as the global optimum $\boldsymbol{\theta}^{*}$.

The output of the MLE is the optimal estimate of unknown model parameters $\boldsymbol{\theta}^{*}$ and will be used directly in the Kalman filter to fuse the displacement and acceleration signals. Multi-rate Kalman filter supplemented by the MLE is expected to provide more accurate estimates of displacement than the isolated multi-rate Kalman filter. In order to evaluate quantitatively the performance of this method, a simulation example of the single degree of freedom (SDOF) oscillation is conducted before the field application.

\section{Numerical validation on simulated data}

The purpose of this section is to access the effectiveness of the proposed data fusion method. This is achieved by examining its performance when applied to simulation data from a single degree of freedom (SDOF) oscillator subjected to an earthquake excitation. The process of applying both MLE and the Kalman filter estimation is demonstrated. Subsequently the results from sole Kalman filter estimation is compared to the results from Kalman filter estimation supplemented by the MLE. Section 3.1 generates the displacement and acceleration signals from a numerical model of a SDOF system. These signals are made more representative of those likely to be encountered in the field by adding white noise to the numerically generated acceleration and displacement signals. Section 3.2 describes the process of applying the MLE to predict the optimal noise parameters for use in the state space model. Finally section 3.3 applies the multi-rate Kalman filter for displacement estimation using several sets of noise parameters and validates that the optimal noise parameters by the MLE provide more accurate displacement estimate than when MLE is not used. 


\subsection{Simulation example of a SDOF system}

This section describes a simulated example of a SDOF system under the earthquake excitation. The measured signals of displacement and acceleration were simulated by contaminating the true responses by a white noise process.

A SDOF oscillator is subjected to the recorded ground motion from the El Centro Earthquake [31]. Equation(11) gives the dynamic motion formula of the system,

$$
m \ddot{x}(t)+c \dot{x}(t)+k x(t)=-m \ddot{x}_{g}(t)
$$

where $m$ is the mass, $c$ is the damping coefficient, and $k$ is the spring stiffness. $\ddot{x}(t), \dot{x}(\mathrm{t}), x(\mathrm{t})$ are the acceleration, velocity and displacement, respectively of the system at time $t$, and $\ddot{x}_{g}(t)$ is the ground acceleration at time $t$. In this simulation, the values of $m, c$ and $k$ used were $m=1 \mathrm{~kg}, c=0.25 \mathrm{~N} \cdot \mathrm{s} / \mathrm{m}$, $k=8 \mathrm{~N} / \mathrm{m}$. The sampling rate of earthquake acceleration signal is $50 \mathrm{~Hz}$.

The first step is to generate the responses of displacement and acceleration of the structural system under the excitation. The ordinary differential equation (ODE) (11) is solved in MATLAB using 'ode 45 ' function to obtain the time histories of displacement, velocity and acceleration. This displacement taken directly from the model is in effect the true displacement which will be used as the reference to evaluate the working performance of the Kalman filter estimation.

To simulate representative measured data, a white noise is superimposed onto the true acceleration and displacement signals. The noise level with $10 \%$ root mean square (RMS) noise-to-signal ratio which is regarded as a normal working range for civil applications is used in this example. The true noise variances of acceleration and displacement measurement are $0.760 \mathrm{~m}^{2} / \mathrm{s}^{4}$ and $0.0064 \mathrm{~m}^{2}$, respectively for the discrete time system. The sample rate is taken as $50 \mathrm{~Hz}$ for measured acceleration and $10 \mathrm{~Hz}$ for measured displacement. The measured signals of displacement and acceleration are shown in Figure 1. The acceleration signal is the upper plot with respect to the y-axis on the left. The displacement signal is the lower plot with respect to the y axis on the right. 


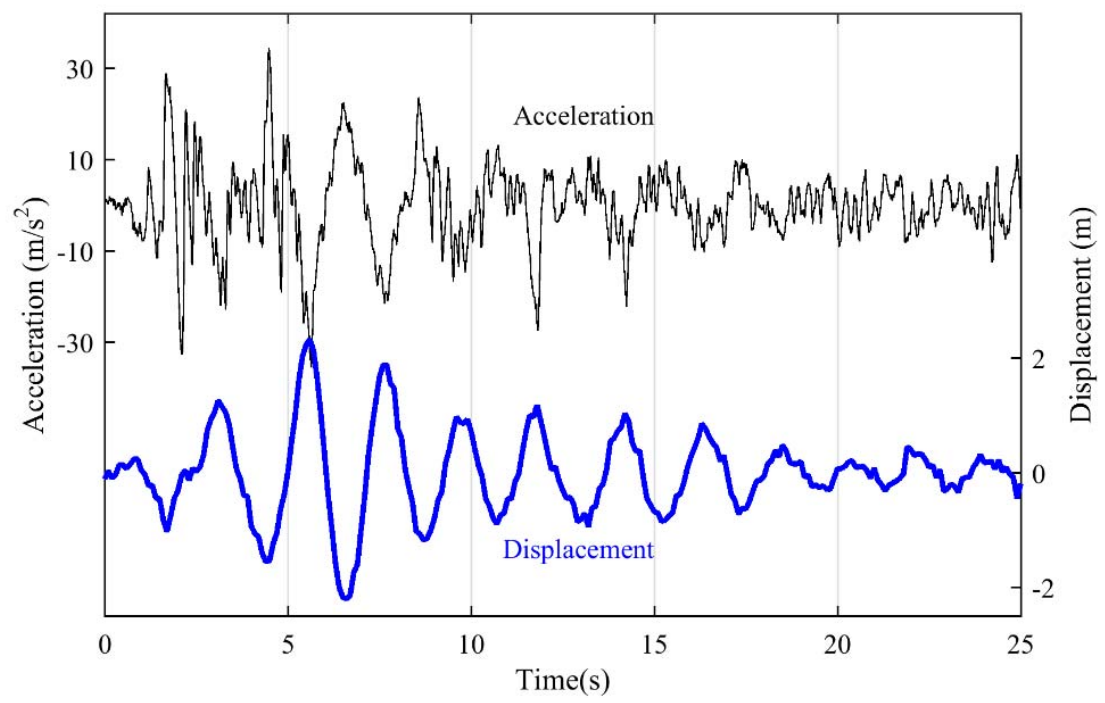

Figure 1 Measured signals of acceleration and displacement of a simulated SOF system under earthquake excitation

\subsection{Parameter estimation by MLE}

This section demonstrates how MLE can be used to estimate the unknown noise parameters which will later be used in the Kalman filter estimation.

Based on the principals in Section 2.2, MLE is used to find the unknown parameters $\boldsymbol{\theta}$ which maximise the log-likelihood function in Equation(9). Given an initial guess $\boldsymbol{\theta}^{0}$ about the unknown parameters, the optimal parameters $\boldsymbol{\theta}^{*}$ are found through the iterations leading to convergence of the log-likelihood function using an optimisation algorithm [32]. Instead of using a single initial guess, ten sets of initial guesses $\left\{\boldsymbol{\theta}_{1}^{0}, \boldsymbol{\theta}_{2}^{0}, \ldots, \boldsymbol{\theta}_{10}^{0}\right\}$ are specified for optimisation separately to derive ten sets of updated parameters $\left\{\boldsymbol{\theta}_{1}^{*}, \boldsymbol{\theta}_{2}^{*}, \ldots, \boldsymbol{\theta}_{10}^{*}\right\}$ by the MLE method. The updated parameters $\boldsymbol{\theta}_{j}^{*}$ corresponding to the maximum log-likelihood after optimisation are taken as the global optimum $\boldsymbol{\theta}^{*}$.

The unknown model parameters $\boldsymbol{\theta}=[q, r, \mathbf{X}(0)]$ include the noise variances $q$ and $r$ corresponding to the acceleration and displacement measurement in continuous-time system as well as the initial states $\mathbf{X}(0)$ of displacement and acceleration. The initial states are specified as zero in all ten sets while the initial values of $q$ and $r$ are generated by the random selection between the specified lower and upper 
bounds. The lower bound for $q$ is selected to be close to zero e.g. $1 \times 10^{-7} \mathrm{~m}^{2} / \mathrm{s}^{3}$ while the upper bound is based on an assumed signal-to-noise ratio (SNR). For example, the variance of acceleration signal shown in Figure 1 is calculated to be $79.31 \mathrm{~m}^{2} / \mathrm{s}^{4}$. Based on the conservative assumption that the SNR value is equal to 0.5 , the variance of noise in acceleration signal has an upper bound of $39.66 \mathrm{~m}^{2} / \mathrm{s}^{4}$. Since the parameter $q$ is the noise variance in continuous-time system, the upper bound of $q$ is derived by dividing the sample rate of acceleration $(50 \mathrm{~Hz})$ reaching $0.79 \mathrm{~m}^{2} / \mathrm{s}^{3}$. Similarly, the lower and upper bounds for the displacement signal are specified as $1 \times 10^{-7} \mathrm{~m}^{2} \cdot \mathrm{s}$ and $0.011 \mathrm{~m}^{2} \cdot \mathrm{s}$, respectively. The initial guesses $\left(q_{j}^{0}\right.$ and $\left.r_{j}^{0}\right)$ are indicated in the second and third columns of Table 1 with units of $\mathrm{m}^{2} / \mathrm{s}^{3}$ and $\mathrm{m}^{2} \cdot \mathrm{s}$, respectively while the updated parameters $\left(q_{j}^{*}, r_{j}^{*}\right.$, initial displacement and initial velocity) are indicated in columns 4 to 7 respectively. Column 9 (Status) indicates if the algorithm converged or not while Column 8 is the updated log-likelihood. It can been seen that set Nos. 2, 6 and 9 failed to converge which was primarily due to problems with the selected initial values. However, set Nos. 1, 3, 4, 5, 7, 8 and 10 all converged to the same parameter values and log-likelihood value. These values are regarded as the global optimum and will be used for data fusion. The noise variances of acceleration and displacement measurement in discrete-time system deduced by the optimal values $q^{*}$ and $r^{*}$ (of the continuous-time system) multiplying the acceleration's sample rate (i.e.

$\left.0.016 \mathrm{~m}^{2} / \mathrm{s}^{3} \times 50 \mathrm{~Hz}=0.80 \mathrm{~m}^{2} / \mathrm{s}^{4}\right)$ are predicted as $0.80 \mathrm{~m}^{2} / \mathrm{s}^{4}$ and $0.0072 \mathrm{~m}^{2}$, respectively. The estimation of measurement noises by MLE is very close to the true values $\left(0.760 \mathrm{~m}^{2} / \mathrm{s}^{4}\right.$ and $0.0064 \mathrm{~m}^{2}$ ) .

Table 1 Updated unknown model parameters and corresponding log-likelihood by MLE

\begin{tabular}{l|ll|lllllll}
\hline & \multicolumn{2}{|l|}{ Initial values $\boldsymbol{\theta}_{j}^{0}$} & \multicolumn{6}{l}{ Updated values $\boldsymbol{\theta}_{\mathrm{j}}^{*}$ found by MLE } \\
\cline { 2 - 8 } Set No. & $q_{j}^{0}$ & $r_{j}^{0}$ & $q_{j}^{*}$ & $r_{j}^{*}$ & Disp $(0)$ & Velo $(0)$ & Log-likelihood & Converge \\
& $\left(\mathrm{m}^{2} / \mathrm{s}^{3}\right)$ & $\left(\mathrm{m}^{2} \cdot \mathrm{s}\right)$ & $\left(\mathrm{m}^{2} / \mathrm{s}^{3}\right)$ & $\left(\mathrm{m}^{2} \cdot \mathrm{s}\right)$ & $(\mathrm{m})$ & $(\mathrm{m} / \mathrm{s})$ & & Status \\
\hline $\mathbf{1}$ & 0.101 & 0.438 & 0.016 & $1.443 \mathrm{e}-4$ & -0.064 & 0.067 & -10.191 & OK \\
\hline $\mathbf{2}$ & $5.340 \mathrm{e}-3$ & $9.634 \mathrm{e}-7$ & $5.340 \mathrm{e}-3$ & $9.634 \mathrm{e}-7$ & 0 & 0 & $-1.388 \mathrm{e} 4$ & Failed \\
\hline
\end{tabular}




\begin{tabular}{l|ll|llllll}
\hline $\mathbf{3}$ & 1.008 & 1.136 & 0.016 & $1.443 \mathrm{e}-4$ & -0.064 & 0.067 & -10.191 & OK \\
\hline $\mathbf{4}$ & 1.003 & $4.996 \mathrm{e}-4$ & 0.016 & $1.443 \mathrm{e}-4$ & -0.064 & 0.067 & -10.191 & OK \\
\hline $\mathbf{5}$ & $1.792 \mathrm{e}-4$ & 0.514 & 0.016 & $1.443 \mathrm{e}-4$ & -0.064 & 0.067 & -10.191 & OK \\
\hline $\mathbf{6}$ & $7.784 \mathrm{e}-3$ & $3.556 \mathrm{e}-7$ & $7.784 \mathrm{e}-3$ & $3.556 \mathrm{e}-7$ & 0 & 0 & $-3.494 \mathrm{e} 4$ & Failed \\
\hline $\mathbf{7}$ & 0.011 & 0.040 & 0.016 & $1.443 \mathrm{e}-4$ & -0.064 & 0.067 & -10.191 & OK \\
\hline $\mathbf{8}$ & $7.751 \mathrm{e}-3$ & $3.157 \mathrm{e}-6$ & 0.016 & $1.443 \mathrm{e}-4$ & -0.064 & 0.067 & -10.191 & OK \\
\hline $\mathbf{9}$ & $1.736 \mathrm{e}-5$ & $4.209 \mathrm{e}-7$ & $1.736 \mathrm{e}-5$ & $4.209 \mathrm{e}-7$ & 0 & 0 & $-4.554 \mathrm{e} 4$ & Failed \\
\hline $\mathbf{1 0}$ & 0.015 & $3.317 \mathrm{e}-5$ & 0.016 & $1.443 \mathrm{e}-4$ & -0.064 & 0.067 & -10.191 & OK \\
\hline
\end{tabular}

\subsection{Estimation of displacement signals by Kalman filter}

This section describes how the multi-rate Kalman filter can be used for estimating displacement. To evaluate the performance of sole Kalman filter estimation versus Kalman filter estimation supplemented by the MLE, several sets of noise parameters including the optimal parameters predicted by the MLE are used for displacement estimation separately. Subsequently, the results obtained are compared to the true response, which allowed the accuracy of sole Kalman filter estimation, and Kalman filter estimation supplemented with MLE to be evaluated.

Based on the mathematical principals mentioned in Section 2.1, the data fusion process involves estimating the mean and error covariance of displacement and velocity through forward Kalman filter and backward smoothing. The estimation could be conducted given the measurement signals of acceleration and displacement, as well as the noise parameters e.g. the noise variances of measured acceleration and displacement. If the sole Kalman filter is applied for the data fusion, the users need to specify the values of measurement noise variances ( $q$ and $r$ ) according to their judgement or experience that might deviate from the true values. Although noise variances $\left(q^{*}\right.$ and $\left.r^{*}\right)$ have been predicted by the MLE in section 3.2, a set of different noise variance values is taken for data fusion to study the effect of parameter selection on the estimation accuracy.

To give the reader a feeling for how far the chosen values for noise variance $q$ and $r$ are from the MLE predicted values $\left(q^{*}\right.$ and $\left.r^{*}\right)$, we define the variables $\eta_{q}$ and $\eta_{r}$ which are the ratios between the selected noise parameters and the prediction by MLE, (i.e. $\eta_{q}=q / q^{*}$ and $\eta_{r}=r / r^{*}$ ). As mentioned in 
[30], the data fusion results (the mean of state variables) remain identical when the values of noise variances ( $q$ and $r$ ) are scaled by the same factor, therefore the possibility of parameter compensation exists for the mean estimation $\mathbf{X}$. For example if values of $q$ and $r$ far from the optimal values of $q^{*}$ and $r^{*}$ are selected such that $\eta_{q}=100$, and $\eta_{r}=100$, the estimated mean of displacement is the same as that from the case when $\eta_{q}=1$, and $\eta_{r}=1$. Similarly this means that the case of $\eta_{q}=1, \eta_{r}=20$ will give the same estimation results about displacement as the case of $\eta_{q}=1 / 20, \eta_{r}=1$.

Due to the above, when investigating the effect of noise parameter selection on the accuracy of the predicted displacement it makes sense to maintain one of the parameters at its optimum value and to vary the other. In this paper the ratio $\eta_{q}$ remains unchanged (equal to 1 ) while the ratio $\eta_{r}$ is varied. Five sets of different noise parameters are chosen, shown in Table 2. The first set is equal to the optimal values determined by $\operatorname{MLE}\left(\eta_{q}=1, \eta_{r}=1\right)$ and the other four sets correspond to the overestimation or underestimation of noise variance in displacement measurement. These five sets of variables are used for Kalman filter estimation separately. The estimation results are compared with the true signals from the simulation to study the estimation accuracy.

Figure 2 indicates the error of four separate displacement signals labelled 'Measurement', 'Estimation (MLE)', Estimation $\left(\eta_{r}=20\right)^{\prime}$ and Estimation $\left(\eta_{r}=100\right)$.

- 'Measurement' is the true displacement signal contaminated with white noise (10\% RMS noiseto-signal ratio),

- 'Estimation (MLE)' is the estimated displacement signal after data fusion of measured displacement and acceleration when the values of $q$ and $r$ are predicted by MLE $\left(\eta_{q}=1\right.$ and $\eta_{r}=1$, essentially this signal corresponds to row 1 of Table 2),

- 'Estimation $\left(\eta_{r}=20\right)$ ' is the estimated displacement signal after data fusion when the values of $q$ and $r$ are defined as $\eta_{q}=1$ and $\eta_{r}=20$ (this signal corresponds to row 2 of Table 2), and

- 'Estimation $\left(\eta_{r}=100\right)$ ' corresponds to row 3 of Table 2. 
It can be seen in the figure that the largest errors occur in the measurement signal whereas ('Estimation (MLE))' signal shows the least errors. 'Estimation $\left(\eta_{r}=100\right)$ ' has less high frequency noise than the 'Measurement' signal, however in a number of areas the amplitude of the errors are of a similar order of magnitude to the errors in the measurement signal. This shows that (i) data fusion brings about improved estimations of displacement; and (ii) using MLE to predict noise variances $q$ and $r$ is likely to result in more accurate estimation of displacement than that by sole Kalman filter where $q$ and $r$ are selected based on judgement/experience. Inaccurate selection of $q$ and $r$ could even lead to displacement estimates that make no accuracy improvement compared with the direct measured signals.

In an effort to further quantify the accuracy of the estimated signals, error analysis is conducted for all the five data sets of Table 2, which is carried out using the normalized root mean square error (NRMSE) defined by

$$
\operatorname{NRMSE}=\frac{\sqrt{\frac{1}{n} \sum_{i=1}^{n}\left(\hat{x}_{i}-x_{i}\right)^{2}}}{\max \left(x_{i}\right)-\min \left(x_{i}\right)}
$$

Where $\hat{x}_{i}, x_{i}$ denote the estimated and true value at time step $i$, and $n$ denotes the number of sampled data points.

The NRMS errors for displacement signals 'Estimation (MLE)', 'Estimation $\left(\eta_{r}=20\right)$ ' and 'Estimation $\left(\eta_{r}=100\right)^{\prime}$ in Figure $2(\mathrm{~b})$ are $0.5 \%, 0.81 \%$ and $1.4 \%$, respectively. Although not plotted in Figure 2 simulations for situations where the noise is underestimated i.e. $\eta_{r}=1 / 20$, and $\eta_{r}=1 / 100$ have been carried out and the associated NRSME values are shown in row 4 and 5 of Table 2. 


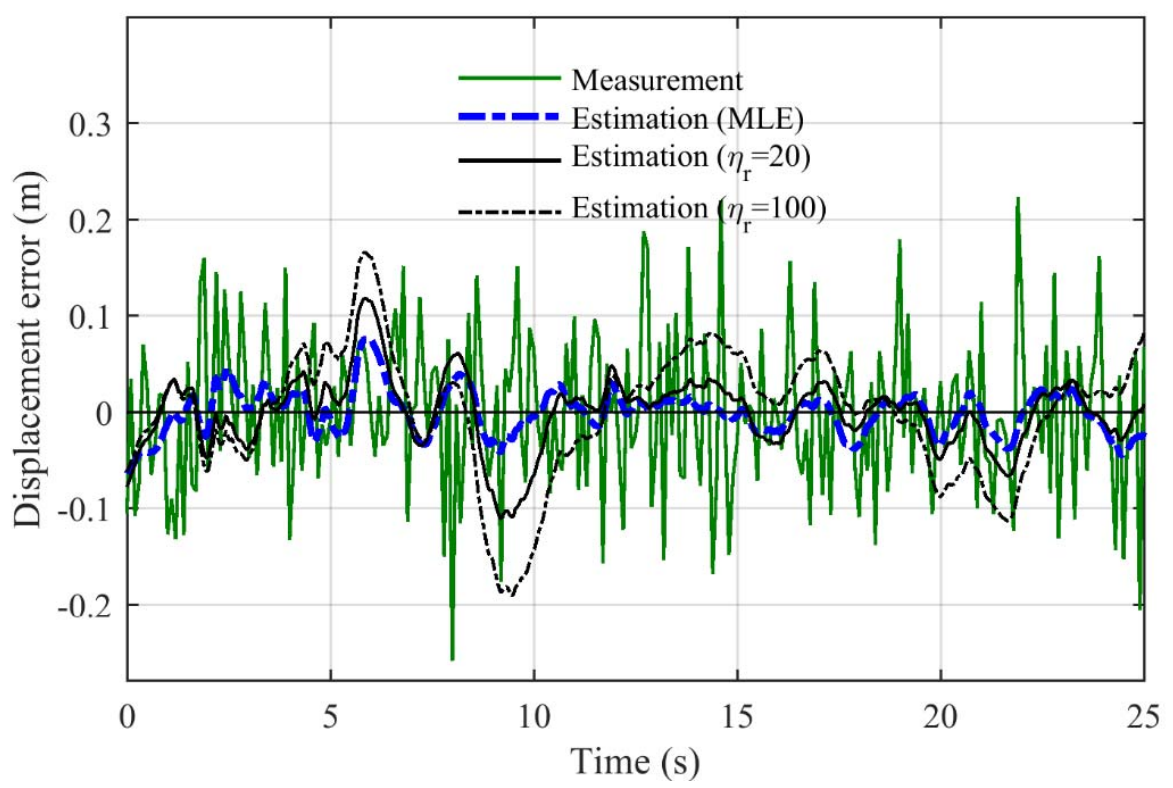

Figure 2 Errors in four displacement signals by comparing with the true one: (i) the measured signal that is the true one superimposed with a white noise (10\% RMS noise-to-signal ratio); (ii) the estimated signal by Kalman filter using the optimal estimate of noise parameters $\left(q^{*}\right.$ and $\left.r^{*}\right)$ by MLE; (iii) the estimated signal by Kalman filter using the noise parameters ( $q=q^{*}$ and $\left.r=r^{*} \times 20\right)$; and (iv) the estimated signal by Kalman filter using the noise parameters $\left(q=q^{*}\right.$ and $\left.r=r^{*} \times 100\right)$.

Table 2 NRMS errors of estimated displacements and velocities by Kalman filter using five sets of noise parameters ( $q$ and $r$ )

\begin{tabular}{lcccc}
\hline Set No. & $\eta_{q}=q / q^{*}$ & $\eta_{r}=r / r^{*}$ & NRMSE of Disp & NRMSE of Velo \\
\hline $\mathbf{1}$ & 1 & 1 & $0.50 \%$ & $0.70 \%$ \\
\hline $\mathbf{2}$ & 1 & 20 & $0.81 \%$ & $0.82 \%$ \\
\hline $\mathbf{3}$ & 1 & 100 & $1.41 \%$ & $0.88 \%$ \\
\hline $\mathbf{4}$ & 1 & $1 / 20$ & $0.50 \%$ & $0.73 \%$ \\
\hline $\mathbf{5}$ & 1 & $1 / 100$ & $0.64 \%$ & $1.05 \%$ \\
\hline Measurement & & & $1.74 \%$ & -- \\
\hline
\end{tabular}


The results in Table 2 and Figure 2 show that the selection of the noise parameters has direct influence on the estimation accuracy of displacement data. Without the step of noise parameter ( $q$ and r) determination (i.e. applying MLE), the estimation accuracy will be highly dependent on the users' selection of parameters. In order to ensure good performance of Kalman filter, MLE is a necessary step to find the accurate prediction of noise parameters. Through this simulation example, the MLE method is validated to be effective for the estimation of noise parameters and beneficial to the estimation performance of Kalman filter. In the next section the same approach is applied to field data.

\section{Field test on Humber Bridge}

Having validated the proposed approach on simulated data, the next step was to implement it on a challenging full-scale structure, in this case the Humber Bridge in UK.

The purpose of this section is to examine the performance of the proposed data fusion method when applied to field data. GPS is the common choice for deformation measurement in long-span bridges [1] however, as discussed in Section 1, GPS performance with respect to accuracy has some shortcomings. Nickitopoulou, Protopsalti, and Stiros [9] suggest standard accuracy of $35 \mathrm{~mm}$ for vertical measurements at 98.5 percentile level, without gross errors such as cycle slip or multipath. A previous short-term monitoring campaign in the Humber Bridge [33] provided a figure of $3 \mathrm{~mm}$ resolution in the vertical direction while a more recent test demonstrates a poorer resolution [1]. Therefore combining GPS signals with accelerometer measurement using data fusion method should be a good approach to overcome the shortcomings of GPS. During the monitoring campaign at the Humber Bridge, the mid-span displacement in vertical direction was estimated by combining the GPS and accelerometer measurement using the multi-rate Kalman filter and MLE. It is noted that the accelerometers Honeywell QA750 used in this application are DC-response devices that enable capturing low frequency motions. The sensor is reported to have a resolution or threshold better than 1 $\mu \mathrm{g}$ while the studies by Brownjohn and Botfield [34] indicated broadband noise floor $(0-100 \mathrm{~Hz})$ at 3 
$\mu \mathrm{m} / \mathrm{s}^{2} / \sqrt{\mathrm{Hz}}$ or better. Piezoelectric accelerometers with no DC response would not be suitable for this method.

In Section 3.3 the true displacement from the numerical model provided the reference for comparing the displacement signals estimated from data fusion. In the field, a reference displacement is required but not readily available. In this study a vision-based system provided a reference as it was felt that this gave the best performance with respect to scanning frequency and measurement accuracy. Measurement accuracy of vision-based system depends on several parameters, e.g. camera-to-target distance, estimation of camera intrinsic parameters, dimension information and dispersion of target tracking results in image, etc. Khuc et al. [35] investigated the measurement accuracy of vision-based system in a short-range distance $(<14 \mathrm{~m})$ and suggested an accuracy of $0.04 \mathrm{~mm}$. Martins et al. [7] demonstrated the uncertainty evaluation of displacement measurement by vision-based system in a long-span bridge monitoring test and illustrated a standard measurement accuracy of $1.7 \mathrm{~mm}$ in the vertical direction. In vision-based system, the nominal resolution of target tracking can be better than 0.01 pixel while the reported accuracy varies from 0.5 to 0.01 pixel [36] which is related to target pattern (texture contrast) [37] and illumination condition. In this application, given the focal length of $300 \mathrm{~mm}$, the camera sensor resolution at $0.0055 \mathrm{~mm} / \mathrm{pixel}$ and the camera-to-target distance at approx. $710 \mathrm{~m}$, the accuracy of 0.1 pixel (artificial target of high-contrast pattern) in image plane corresponds to an accuracy (or rather resolution) of $1.3 \mathrm{~mm}$ in the structural system. However, the vision-based system meets several challenges on site e.g. target pattern change due to lighting condition, camera shake caused by human or wind and partly obstructed view. On the day of the test, environmental conditions were optimal for camera monitoring (calm day, stable light conditions) and from previous experience of using the system in these conditions it is believed to reflect true displacement with good accuracy. In the text which follows, this section is broken into three parts, Section 4.1 describes the different sensing systems used during the test, Section 4.2 reports the performance of the proposed approach when applied to field data and Section 4.3 gives a brief overview on the challenge of varying measurement noise in the displacement signal returned by GPS measurements. 


\subsection{Test configuration and sensing systems used}

The Humber Bridge, opened in 1981, has a main span of $1410 \mathrm{~m}$ and side-spans of $280 \mathrm{~m}$ and $530 \mathrm{~m}$ linking the small towns of Hessle and Barton in UK. The deck is formed using aerodynamic steel box sections which are $22.5 \mathrm{~m}$ wide and $4.5 \mathrm{~m}$ deep with $3 \mathrm{~m}$ walkway cantilevers each side (See Figure 3(f)). A structural health monitoring system was installed in the Humber Bridge in September 2010

[1]. It includes a GPS base station at the bridge tower, two GPS rovers (Leica GMX902) mounted on the main cables at mid-span and three QA750 accelerometers mounted inside the steel box girder at mid-span (two vertical and one lateral/horizontal) [1]. The GPS rover can be seen mounted on the main cable in Figure 3(b) and is also indicated in the schematic in Figure 3(f). Figure 3(c) shows the lateral accelerometer and one of the vertical accelerometers in the east side inside the deck. The position of these accelerometers is also indicated in the schematic in Figure 3(f). Ideally the vertical accelerometer would be in the same position transversely as the GPS rover, however it is close enough that any errors due to not being co-located will be small.

A single day of field test using the vision-based monitoring system was performed on $22^{\text {nd }}$ July 2015 to measure the displacement at mid-span of the bridge. The vision-based monitoring system used in the test was developed by Imetrum Limited, UK. It consists of one GigE high performance camera and a controller containing the video processing package for video acquisition and real-time analysis. The tracking algorithms adopted are correlation-based template matching and super resolution techniques [38]. The planar homography based on dimension correspondences in image plane and structural surface plane are adopted to transform the image coordinates (i.e. pixel) to structural coordinates (i.e. mm). The output includes the two-dimensional translations along vertical and transverse directions of the bridge.

The camera was equipped with the lens of $300 \mathrm{~mm}$ focal length mounted on a tripod at the base of the north tower as shown in Figure 3(d). Further information on the camera set up is in Figure 3(e) that shows the camera looking south toward the mid-span of the bridge, about $710 \mathrm{~m}$ from the camera. The system is operated using the laptop and Imetrum controller that can be seen on the table in Figure 3(e) and the system was powered using the portable battery pack under the table. Essentially the camera is 
zoomed in on the artificial target of concentric rings which has been mounted in a $1 \mathrm{~m} \mathrm{x} 1 \mathrm{~m}$ metal frame attached to the parapet on the east side of the bridge and is shown in Figure 3(b). The purposes of installing the artificial target were twofold, 1) improving the tracking stability and 2) providing the reference of geometric information for the determination of the planar homography matrix. The positions of target and GPS rover along the transverse direction are illustrated in Figure 3(f). Figure $3(\mathrm{~g})$ shows the view from the camera and the target is visible in the bottom left of the image. 
(a)

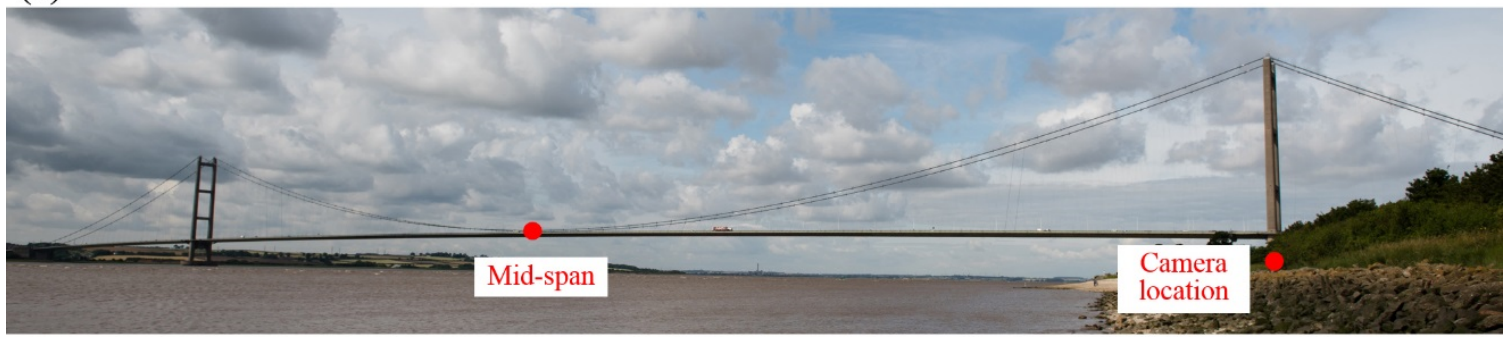

(b)

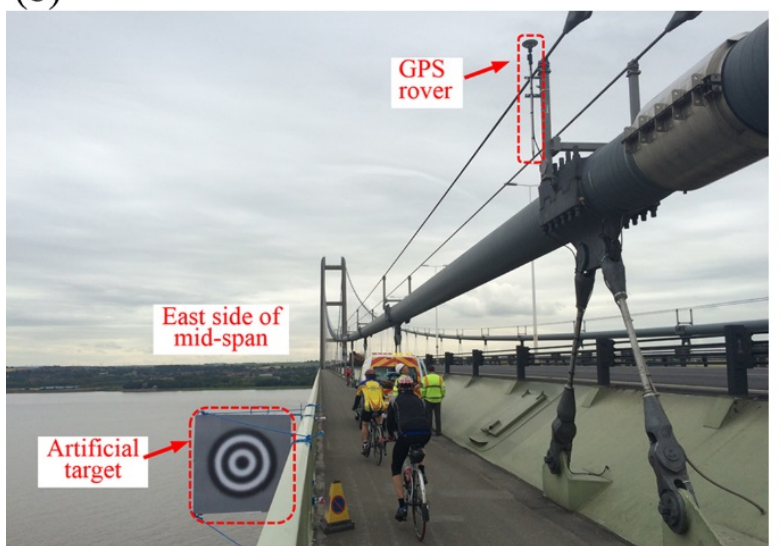

(c)

(d)

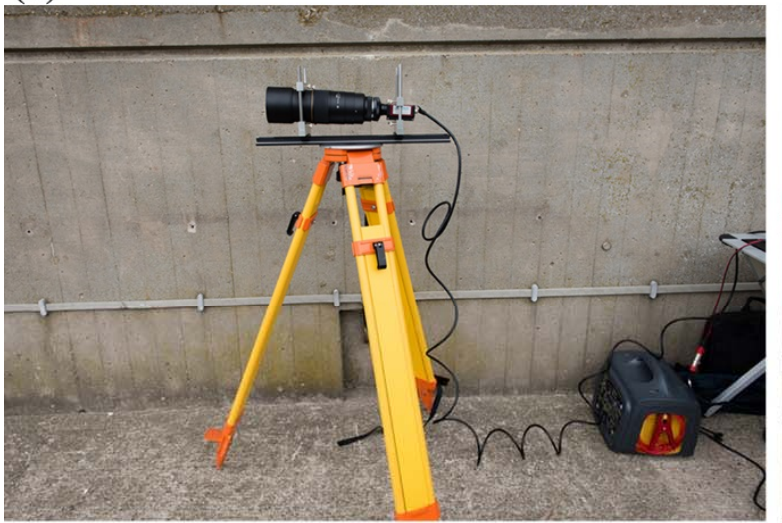

(f)

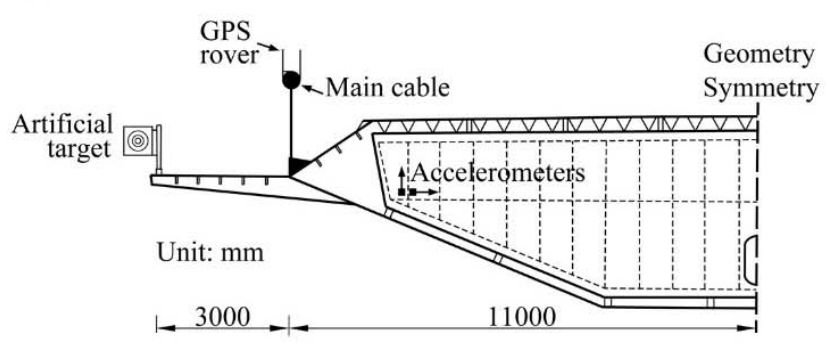

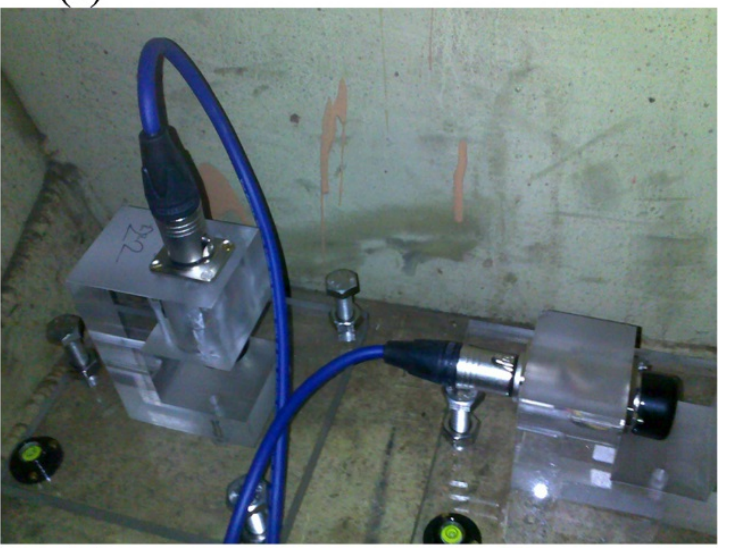

(e)

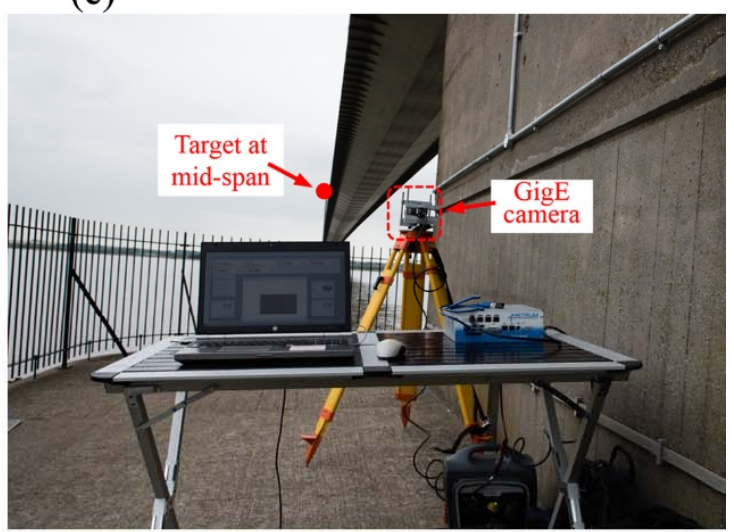

(g)

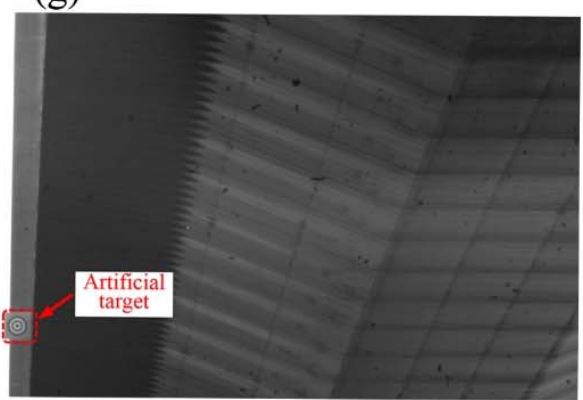

Figure 3 Configurations of measurement sensors during the Humber Bridge test: (a) east elevation of the bridge; (b) GPS rover and artificial target at east side of mid-span; (c) two accelerometers inside the girder at mid-span; (d) side elevation of the camera/tripod; (e) camera set up on east side of north tower, camera looking south toward target at mid-span; (f) configuration of girder section (east side); and (g) target/mid-span of the bridge when viewed through the camera. 
The sample rates of GPS and QA accelerometers in the long-term monitoring system are $1 \mathrm{~Hz}$ and 20 $\mathrm{Hz}$, respectively and the frame rate used for the vision-based system was $10 \mathrm{~Hz}$. These three sensing systems had independent data acquisition systems using different clocks and the data are required to be time-aligned before the data fusion and measurement comparison. The time shift among these three sensing systems was corrected by finding the maximum of cross correlation of three signals (all interpolated to $20 \mathrm{~Hz}$ ), the vertical displacement by GPS, the vertical displacement by vision-based system and the double-integrated displacement with base correction using vertical acceleration data. The issue of synchronising data streams was studied in a modal survey of Humber Bridge [39]. It was found that for vibration model up to $1 \mathrm{~Hz}$ (1 second period), a synchronisation error of $0.007 \mathrm{~s}$ did not compromise modal analysis. The focus in this study is on the quasi-static responses of the bridge and fundamental vibrations modes having periods of at least $8 \mathrm{~s}(0.117 \mathrm{~Hz}$ for the first modal frequency). Therefore, the synchronisation error (less than $0.05 \mathrm{~s}$ ) on the data fusion is judged to be acceptable.

To illustrate the type of load causing the displacements shown later in this section, a Nikon D800 DSLR camera (positioned a few hundred metres east of the bridge) was used to video-record traffic on the bridge. Figure 4(a) is one frame captured from the recorded video when two heavy goods vehicles (HGVs) were approaching the mid-span from opposite directions. Figure 4(b) shows the corresponding measurement by the camera system in vertical direction, with vertical deflection at mid-span caused by the two vehicles reaching $221 \mathrm{~mm}$. This figure allows the reader to visualise the magnitude of the loads causing a particular level of displacement. 
(a)

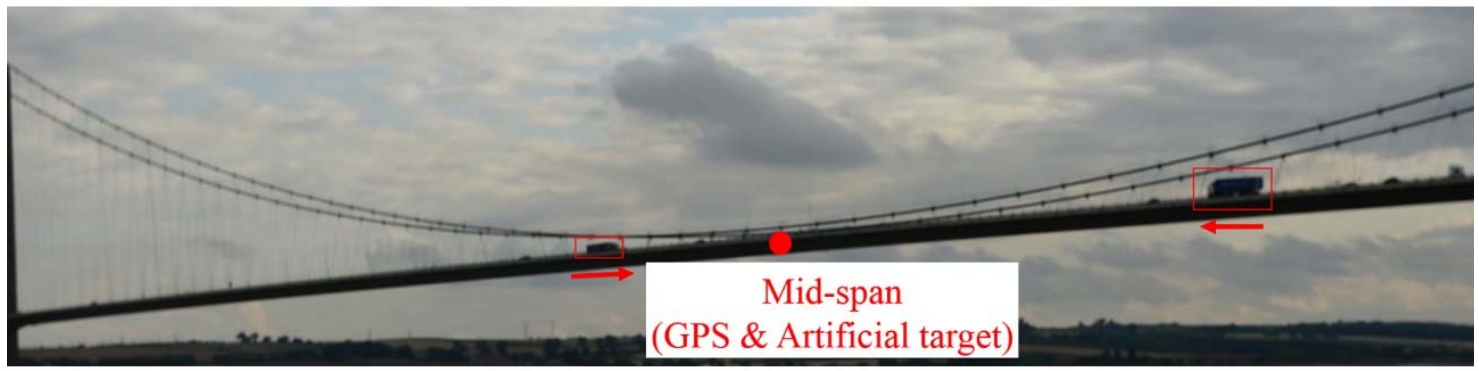

(b)

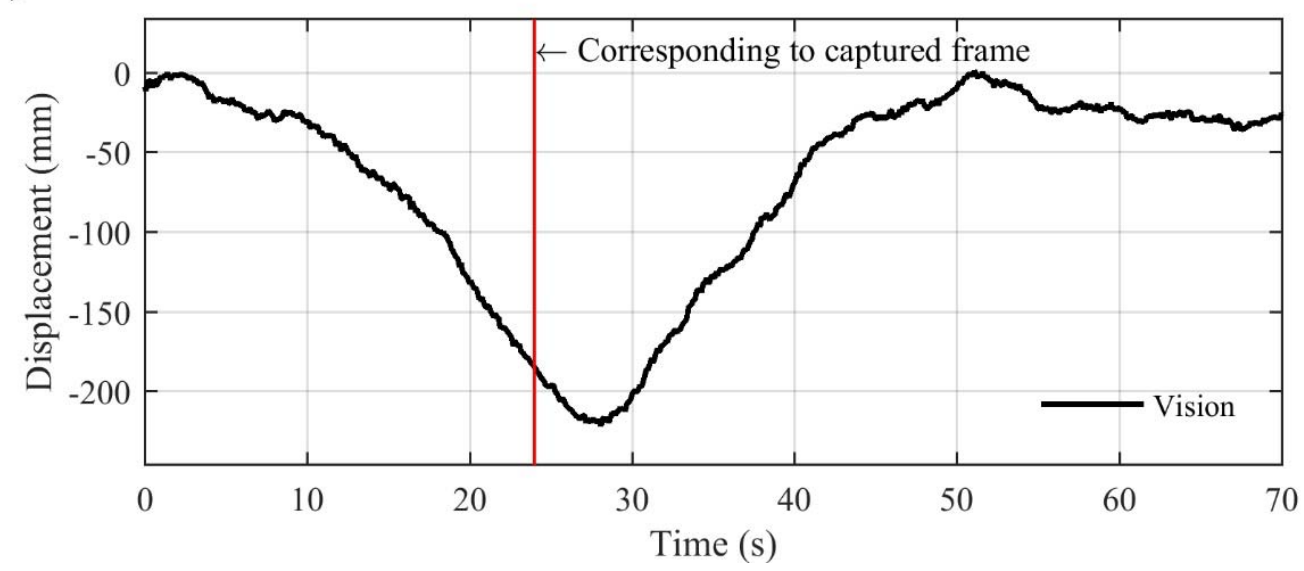

Figure 4 Measurement by vision-based system when two HGVs were approaching the mid-span: (a) captured frame from a video; and (b) vertical displacement of bridge at mid-span measured by visionbased system.

\subsection{Examining performance of data fusion approach}

This section reports the results of applying the proposed MLE enhanced data fusion method to the field data recorded on Humber Bridge and for convenience it is broken into three subsections. Section 4.2.1 describes the input signals and the displacement signal estimated following MLE and data fusion. Section 4.2.2 briefly demonstrates the 'extra' information on the dynamic response of the structure contained in the estimated displacement signal. Finally section 4.2 .3 compares GPS originating displacement signals (raw and estimated) to the benchmark displacement signal measured by the vision-based system. 


\subsubsection{Applying MLE and Kalman filter to field data}

In this section, the data fusion method proposed in Section 2 was adopted to merge the vertical displacement from the GPS sensor with the collocated acceleration measured by QA accelerometer.

The quasi-static displacement in vertical direction induced by traffic loads is the main interest of this study. Ten-minute signals of vertical displacement by GPS, vertical displacement by vision-based system and vertical acceleration from QA accelerometer are truncated shown in Figure 5, where acceleration and displacement are plotted with respect to the left and right y-axes, respectively. It can be seen in the figure that broadly speaking the GPS displacement agrees very well with the visionbased displacement, this is evidenced by the fact that cross correlation between two displacement signals is $98.4 \%$. For the scale used in this plot it is difficult to accurately quantify the magnitude of the differences between the two signals but in some places it looks to be in the region of 10-20 $\mathrm{mm}$. Further information on the differences between the two signals is provided later in Figure 9.

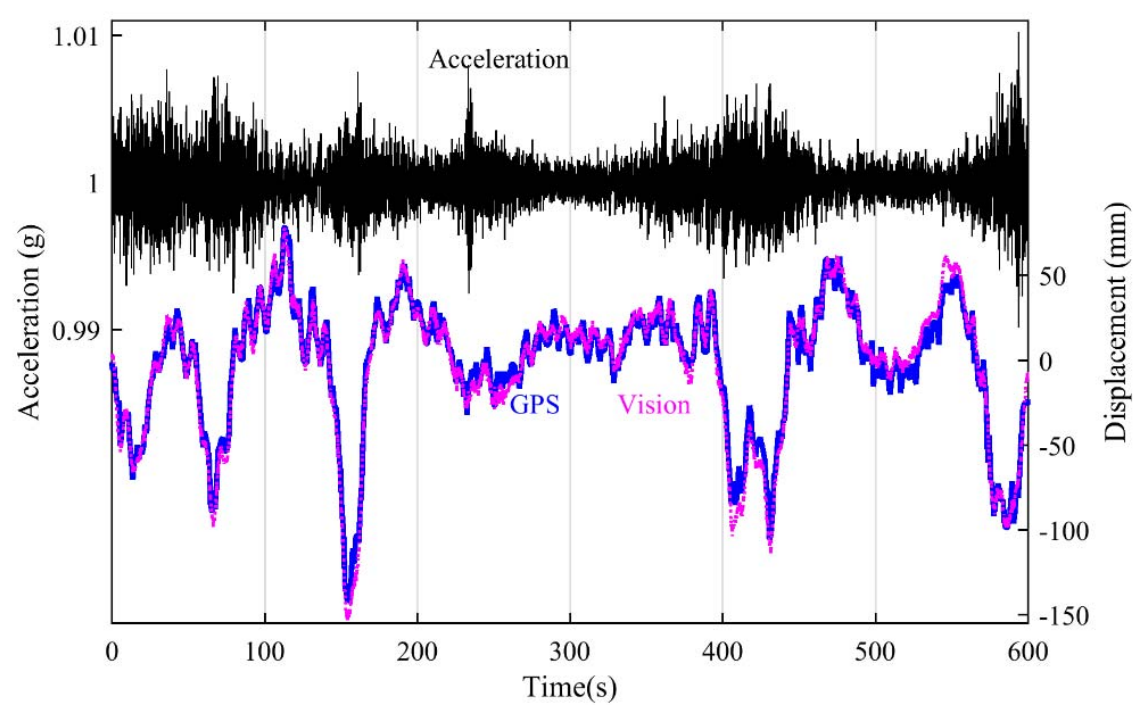

Figure 5 Measured signals by accelerometer, GPS and vision-based system in vertical direction in the Humber Bridge

The first step of analysis is to identify the noise parameters in GPS and accelerometer measurement using the MLE. The noise in GPS and QA accelerometer measurement is assumed to be a white noise process with zero mean and fixed variance and then the MLE proposed in section 2.2 is used to 
estimate the noise variances of these two signals. The estimation process (e.g. selecting the initial values for unknown parameters, updating the values for unknown parameters based on an optimisation algorithm and then finding the global optimum) was similar to that for the numerical example presented in section 3.2 and only the estimation results are demonstrated here. The results of the MLE provide estimates of standard deviations of GPS noise and accelerometer noise in vertical direction as $6.79 \mathrm{~mm}$ and $1.77 \mathrm{~mm} / \mathrm{s}^{2}$, respectively (for the signals shown in Figure 5). These results will be adopted in the Kalman filter estimation in the next step.

The second step of analysis is to fuse the GPS and accelerometer measurement in vertical direction by multi-rate Kalman filter algorithm. Prior to fusion, the acceleration is scaled from $\mathrm{g}$ (see Figure 5) to $\mathrm{mm} / \mathrm{s}^{2}$. The variation between GPS measurement and down-sampled estimation is regarded as GPS noise and is indicated in Figure 6 with the RMS value of $6.45 \mathrm{~mm}$.

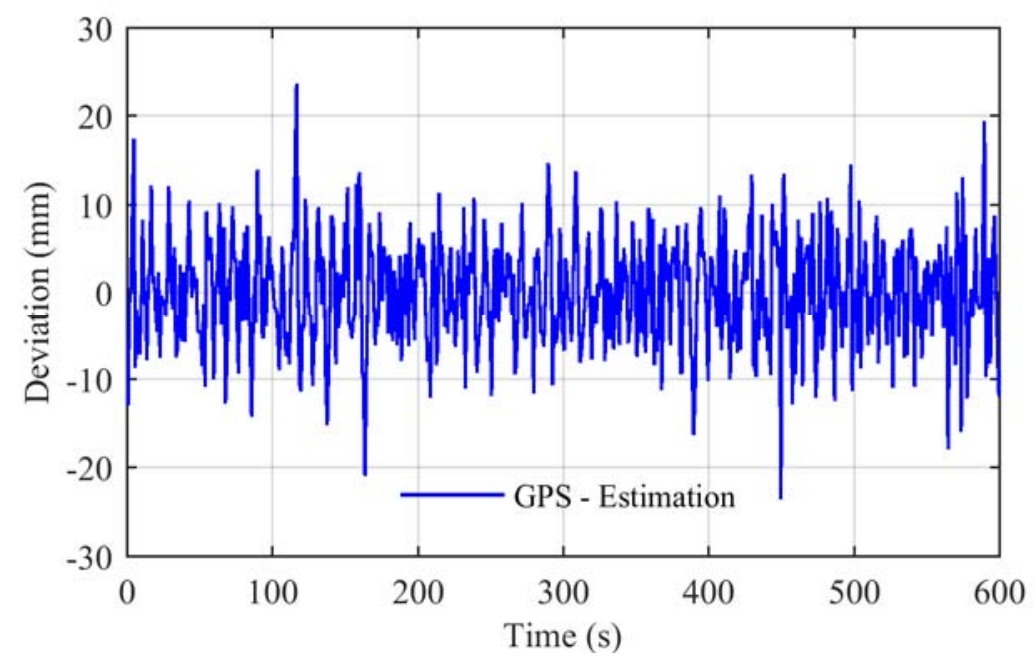

Figure 6 Difference between two displacement time history signals: GPS direct measurement and Kalman filter estimation using the optimal estimate of noise parameters by MLE.

In the following sections (4.2.2 and 4.2.3), the estimation results by Kalman filter and MLE will be evaluated in frequency domain compared with the measurement by accelerometers and in time domain compared with the measurement by vision-based system. 


\subsubsection{Enhanced dynamic information in displacement estimate following data fusion}

The power spectral densities (PSD) of the raw acceleration data, the raw GPS displacement signal and the estimated displacement (following data fusion) were obtained by Welch's method [40]. The data were split into 10 equal-length segments with $50 \%$ overlap, and each segment was windowed with a Hamming window and the results are shown in Figure 7. From the previous studies on the bridge it is known that (vertical) modal frequencies exist at $0.117 \mathrm{~Hz}, 0.31 \mathrm{~Hz}$ and $0.46 \mathrm{~Hz}$ [41]. The GPS system on Humber Bridge has a sample rate of $1 \mathrm{~Hz}$ [1], which means the sample rate is sufficiently high to capture modal frequencies in the range $0-0.5 \mathrm{~Hz}$. If the GPS plot in Figure 5 is examined, it can be seen that the first vertical modal frequency at $0.117 \mathrm{~Hz}$ is identified by GPS measurement, however two other modal frequencies which occur in the $0-0.5 \mathrm{~Hz}$ range (namely $0.31 \mathrm{~Hz}$ and $0.46 \mathrm{~Hz}$ ) are not detected. In theory, the GPS measurement has the chance to capture the second and third vertical modes but in fact it has failed to do so. Similarly, the measurement by vision-based system with the sample rate at $10 \mathrm{~Hz}$ identifies the first and second modal frequencies $(0.117 \mathrm{~Hz}$ and $0.31 \mathrm{~Hz})$ and fails to capture more.

Although both GPS and vision-based system have been validated to be feasible for structural dynamic monitoring [14],[42], it is difficult in this case to use the displacement sensing system to measure the structural dynamics by providing a high sample rate. This is mainly because the displacement induced by vehicle loads is always dominated by the static and quasi-static components while the dynamic component of displacement is relatively small (i.e. the root mean square of de-trended acceleration signal during this time interval is only $0.016 \mathrm{~m} / \mathrm{s}^{2}$ ) and easily contaminated by the measurement noise.

In the estimated displacement fused by GPS and accelerometer measurement, the modal frequencies with the value $0.31 \mathrm{~Hz}$ and $0.46 \mathrm{~Hz}$ are clearly identified in Figure 7. This indicates that the noise in estimated displacement is reduced making the dynamic components clear. The sampling rate of the estimated displacement signal is $20 \mathrm{~Hz}$ and so, it also proved possible to identify the modal frequency at $0.63 \mathrm{~Hz}$. 


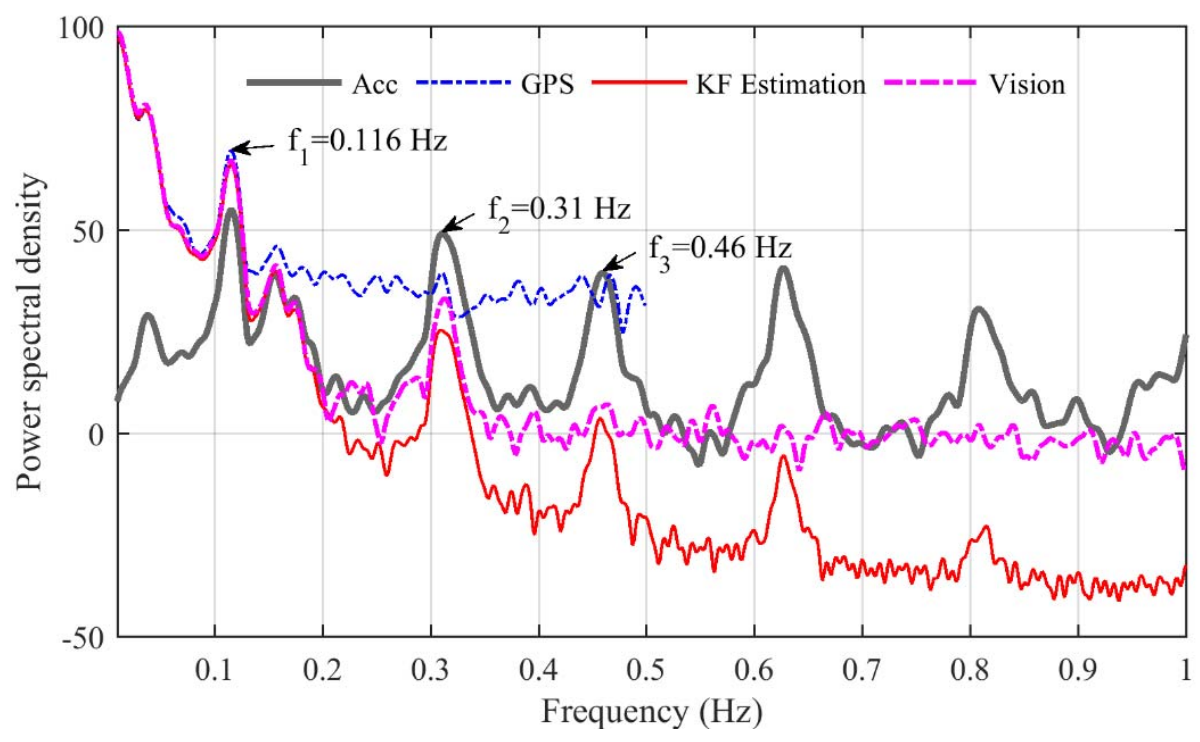

Figure 7 Power spectral densities of four time history signals by Welch's method: (i) the raw acceleration signal; (ii) the raw GPS displacement signal; and (iii) the estimated displacement by fusing the acceleration and displacement measurement using Kalman filter; and (iv) displacement signal measured by vision-based system.

\section{$\underline{\text { 4.2.3 Comparing raw GPS and estimated displacements to benchmark vision-based displacement }}$}

The measurement by the vision-based system (Imetrum) was adopted to evaluate the estimation results. Before deploying the Imetrum system on Humber Bridge, the system accuracy and stability have been validated through field tests on short-span bridges in Exeter by comparing with the measurement obtained by contact sensors (i.e. LVDT) [43]. Therefore the measurement by visionbased system is believed to a suitable benchmark to compare with GPS measurement and estimation by data fusion.

If Figure 3(f) is examined it can be seen that the artificial target, GPS rover, and vertical accelerometer were not at the same transverse position on the deck. The artificial target is mounted on the parapet, the GPS rover is mounted on the main cable $3 \mathrm{~m}$ from the parapet and the accelerometer is on the inside of the box girder a further $2.5 \mathrm{~m}$ from the parapet. Therefore it is necessary to compensate for the influence of torsional deck rotation/tilt before any comparison. Since the deck rotation under traffic loads is dominated by quasi-static components with very small dynamics, the influence of location deviations 
on vertical acceleration measurement could be ignored while the influence on vertical displacement measurement should be considered. The GPS rover was mounted at the top of main cable, $11 \mathrm{~m}$ away from the centre of box section. The artificial target was located at the edge of walkway cantilevers, about $14.5 \mathrm{~m}$ away from the centre. Only the quasi-static tilt of the deck was considered and corrected which was estimated by applying low-pass filter to the height difference of two GPS rovers located at mid-span (one rover on each of the two main cables). The filtered result is validated by cross checking it with the rotation angle of deck predicted from low frequency component of lateral acceleration shown in Figure 8 which senses rotation via the component of gravity resolved along the DC accelerometer axis. The moving trends of two predictions look similar and the GPS height variation after low-pass filter was adopted for tilt correction. Assuming that the deck rotates around the centre as a rigid body, the tilt was calculated by the rotation angle and the distance to deck centre. The tilt difference between vision-based system and GPS measurement is then removed from the raw measurement.

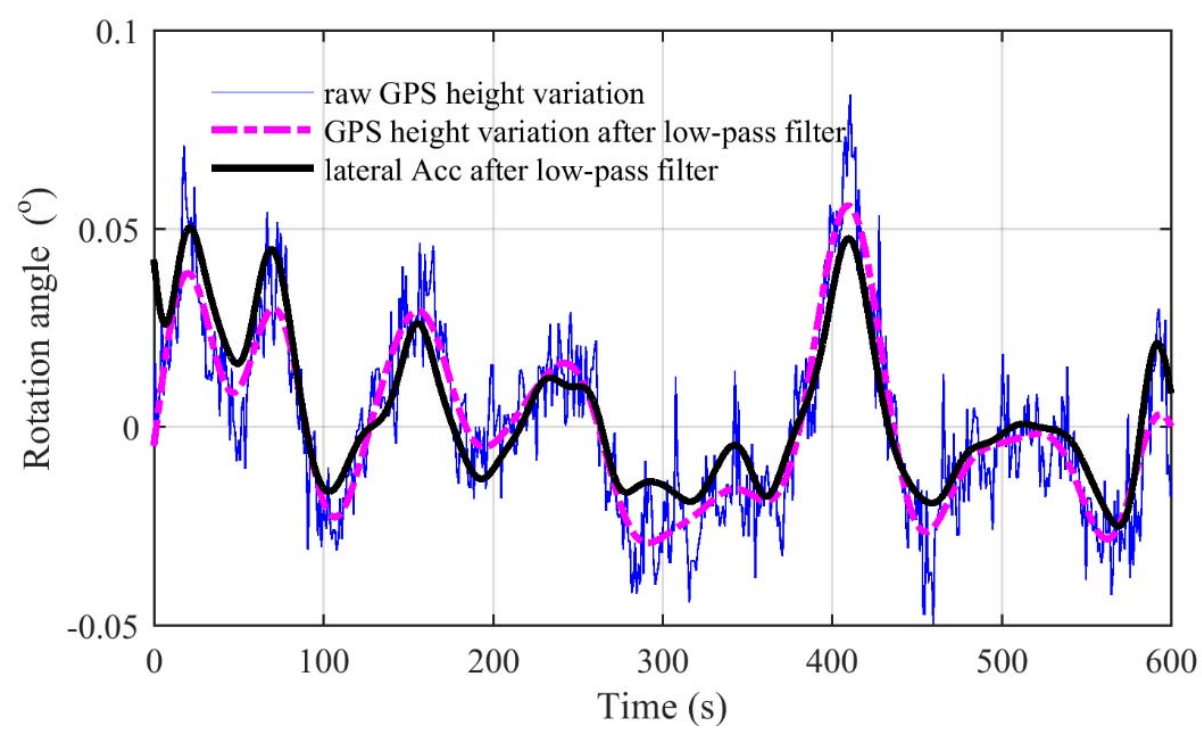

Figure 8 Predicted rotation angle of deck in the longitude direction at mid-span by: (i) variation of the two GPS height measurement at two sides of mid-span; (ii) low-pass filter results of variation of the two GPS height measurement (cut-off frequency at $0.02 \mathrm{~Hz}$ ); and (iii) DC component of lateral acceleration measurement at mid-span by low-pass filter (cut-off frequency at $0.04 \mathrm{~Hz}$ ).

In this section three different displacement signals originating from GPS data are compared to the benchmark vision-based displacement signal: the raw GPS measurement (GPS), and Kalman filter 
estimation using two different values of $\eta_{r}$ (Estimation $\eta_{r}=1$, and Estimation $\eta_{r}=200$ ). Here $\eta_{q}$ and $\eta_{r}$ have the same meaning as in Section 3.3, and for both estimated displacement signals $\eta_{q}=1$. The purpose of using two different values of $\eta_{r}$ is to demonstrate the difference in the estimated displacement when noise parameters are well or poorly selected. The results of these three displacement signals subtracted by the optical measurement (down-sampled to equal sample rate as GPS measurement) are shown in Figure 9.

It can be seen that the largest errors occur in the 'GPS' measurement signal, Estimation $\left(\eta_{r}=1\right)$ shows the least errors, and Estimation ( $\left.\eta_{r}=200\right)$ has errors somewhere in between. (Note, 'GPS' data points in the figure are in effect the differences between the two displacement signals shown earlier in Figure 5 after the difference in the transverse position of the two sensors is corrected for). The errors evident in Figure 9 can be summarised as normalised root mean square error (NRMSE) values as defined in Equation(12). The NRMS error of displacement signal for GPS measurement, Estimation $\left(\eta_{r}=1\right)$ and Estimation $\left(\eta_{r}=200\right)$ are $3.17 \%, 2.37 \%$ and $2.90 \%$, respectively. The results show that using data fusion results in displacement signals that are more accurate than the raw GPS displacement signal with the NRMS error decreased from $3.17 \%$ to $2.37 \%$, and moreover that using MLE to identify the optimal estimate of noise parameters results in a more accurate displacement estimation than that when noise parameters are specified based on subjective judgement.

As a final comment on Figure 9, it is evident that there is some low-frequency variation between the GPS originating signals and the vision-based measurements. This might be caused by the difference of sensor locations, the measurements from two sensors are not perfectly correlated to the deck movement i.e. there might be some local movements in the main cable where GPS rover was mounted or in the parapet where the artificial target was attached. 


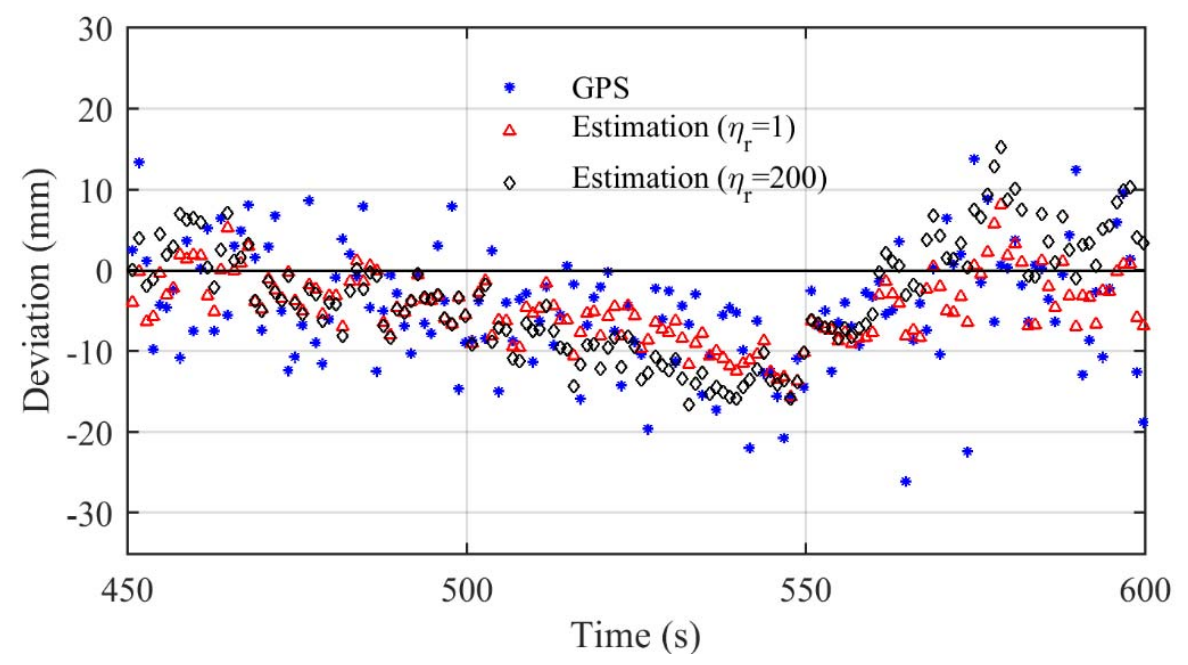

Figure 9 Comparison of three displacement signals with the measurement by vision-based system: (i) GPS raw measurement; (ii) Kalman filter estimation using the optimal noise parameters $\left(q^{*}\right.$ and $\left.r^{*}\right)$ predicted by MLE; and (iii) Kalman filter estimation using the noise parameters ( $q=q^{*}$ and $\left.r=r^{*} \times 200\right)$.

\subsection{Daily variation of noise in GPS measurements}

Having seen in Figure 9 the errors that can result in the estimated displacement signal due to incorrectly estimating the noise present in the raw GPS displacement measurements, this section examines how the GPS noise varies over the course of the day. The fact that the noise in GPS measurements is not constant means that if using GPS measurements in a data fusion system it is necessary to update the values of $q$ and $r$ at certain time intervals using MLE. It is noted that the noise parameter estimation step using MLE is not a real-time processing and instead is to deal with offline data. The text below describes the variation in the noise characteristics of the raw GPS measurement observed on the day of the test at Humber Bridge.

The positioning accuracy of GPS measurement depends mainly on the atmosphere condition, satellite geometry and the possible secondary reflection or multi-path [9]. Therefore the GPS noise should be time dependent even during a single day, since the satellites are not geo-stationary. To investigate the variation in GPS noise over the course of the day, two ten-minute signals of GPS and QA 
accelerometer measurement in vertical direction were truncated for data fusion. The first time interval was from 12:35 to 12:45 and the second one was from 18:50 to 19:00. The measurement signals after time synchronisation are shown in Figure 10(a) and (b) respectively.

Firstly, the MLE method was applied to estimate the noise parameters of GPS and accelerometer measurement for both time intervals. The result indicates that the standard deviations of GPS noise were estimated as $7.28 \mathrm{~mm}$ and $13.70 \mathrm{~mm}$ for time intervals 1 and 2 respectively. The GPS measurement in the second time interval (18:50 to 19:00) is noisier than in the first one, i.e. it has a larger standard deviation of noise.

To allow the larger noise of interval two to be visualised, the estimated noise parameters are used for data fusion by the Kalman filter and the resulting estimated displacements subtracted from the raw GPS measurements are shown in Figure 10(c) and (d) as approximate GPS measurement noise. The RMS of estimated noise was $7.10 \mathrm{~mm}$ and $13.36 \mathrm{~mm}$, respectively. The signals in Figure 10 show that the GPS signal in the second time interval is much noisier than the first time interval and that the noise variance of GPS measurement varies with time.
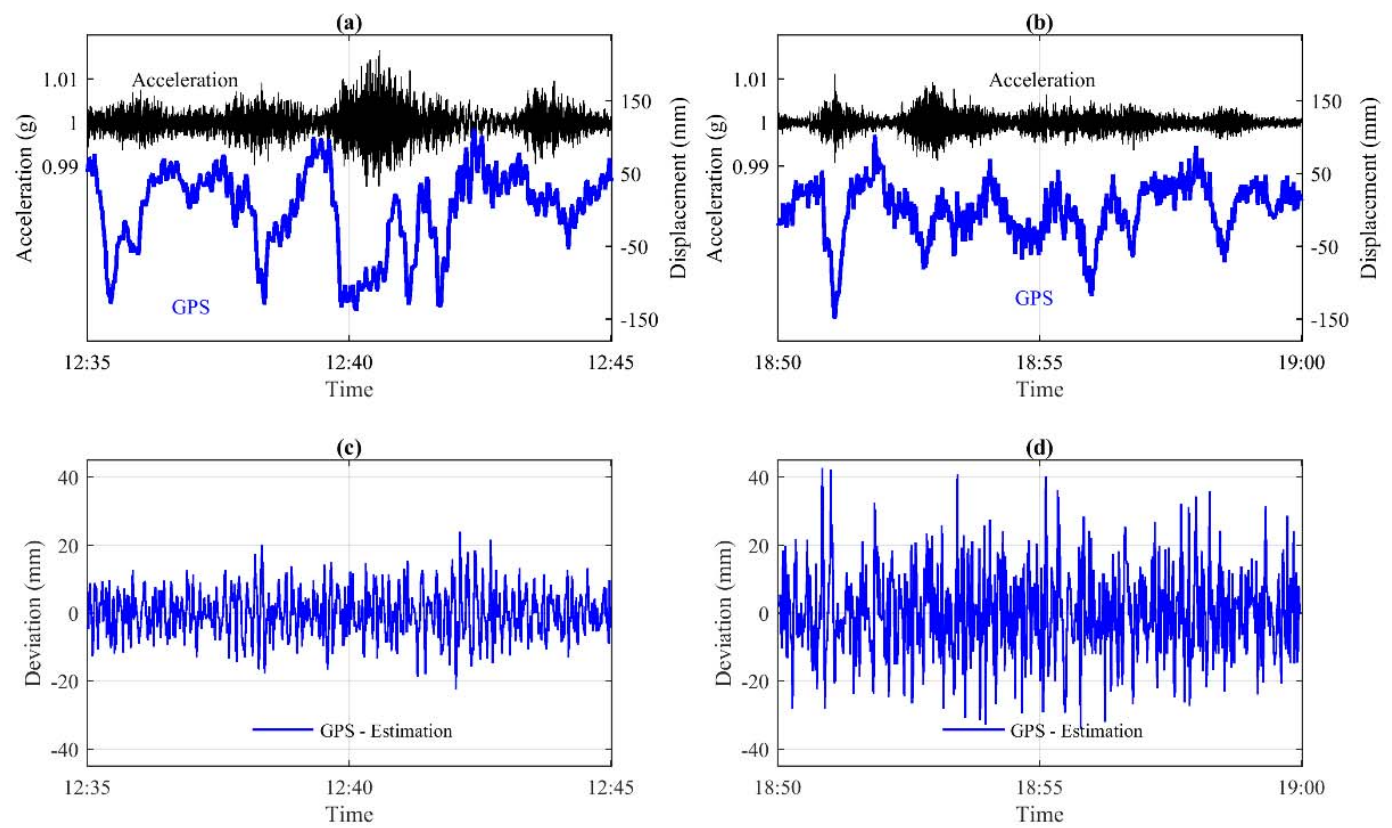
Figure 10 Variation of GPS noise with time: (a) raw acceleration and displacement signals for time interval 1; (b) raw acceleration and displacement signals for time interval 2; (c) approximate GPS noise for time interval 1; and (d) approximate GPS noise for time interval 2.

Having seen in Figure 10 the time-dependent nature of GPS noise, it deserves attention to deal with the long-time signals using the proposed data fusion method. In the Kalman filter, the GPS noise is assumed as a Gaussian process with zero mean and fixed variance while the value of GPS noise variance is pre-determined by the MLE using offline measurement data. For a robust application of this method over longer time e.g. several hours or days, it is suggested to update the GPS noise parameters every half hour e.g. truncating the first five minutes of 30-min data for noise parameter estimation using the MLE and applying the tuned noise parameters for data fusion of the whole 30min signals.

Since the number of available satellites have impact on the GPS positioning accuracy, Figure 11(a) provides the time-dependent characteristics of available satellite over the whole day. It is observed that the number of available satellites from 05:00 AM to 11:30 AM decreased to 3 which are not enough for 3D positioning. Therefore the GPS displacement data during this period was not available. Based on the available GPS data, the MLE was applied to estimate the noise parameters during the data fusion of GPS and accelerometer measurement in vertical direction. The estimated results are shown in Figure 11 (b) with the standard deviation of GPS noise varying from $6.16 \mathrm{~mm}$ to $16.19 \mathrm{~mm}$. 

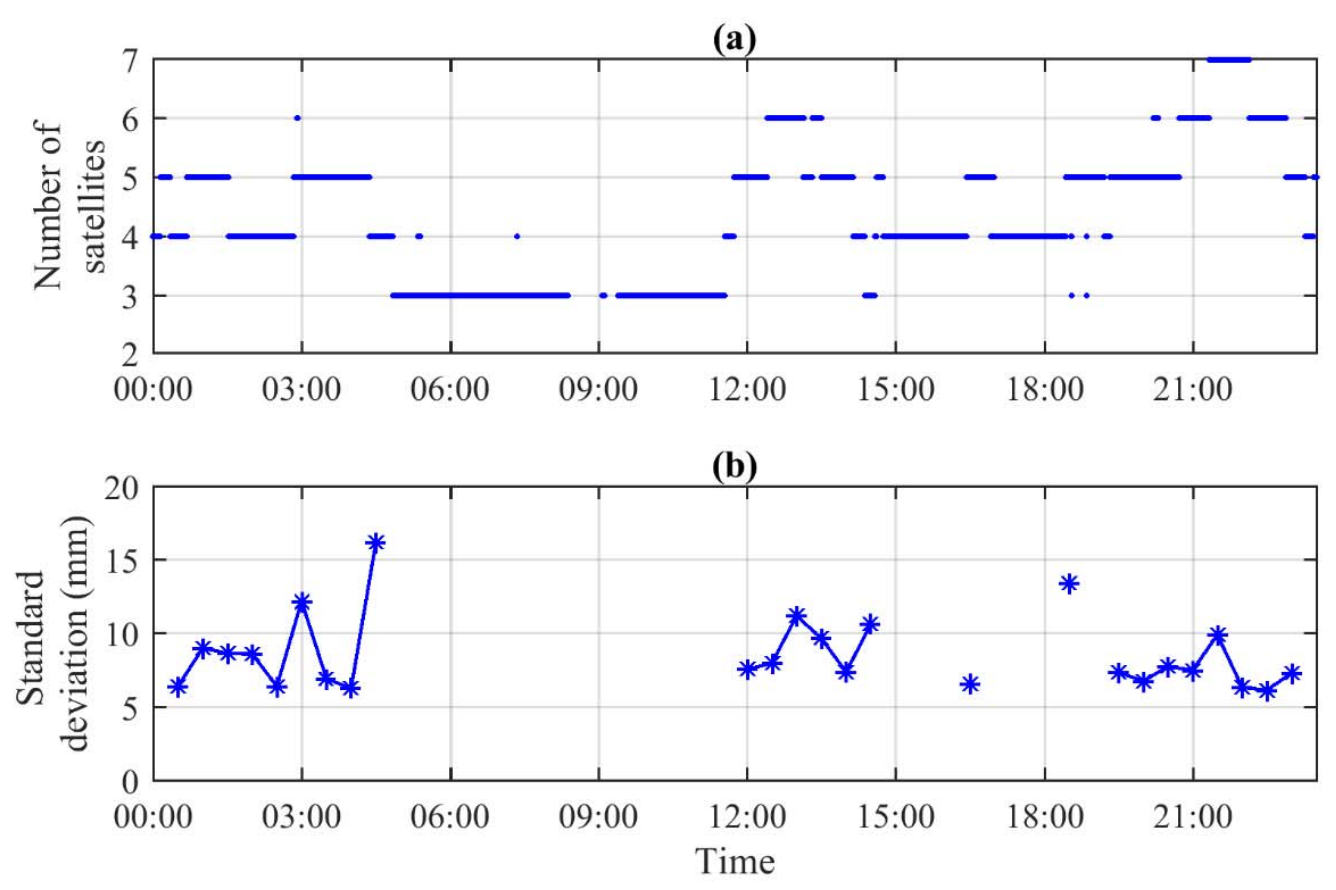

Figure 11 Characteristics of GPS measurement over the whole day: (a) number of available satellites; and (b) estimation results of standard deviation of GPS noise by the MLE.

\section{Conclusions}

Data fusion of displacement and collocated acceleration signals using Kalman filter and maximum likelihood estimation is validated to be effective for improving displacement accuracy. MLE is effective at accurately determining the noise parameters of measurement signals which are required by the application of Kalman filter. Incorporating the MLE could overcome the limitations of parameter selection according to users' judgement and ensure the good performance of Kalman filter.

The data fusion method is also practical to field implementations. The results in the Humber Bridge test indicate the effectiveness to improve the GPS accuracy and to widen the frequency bandwidth of displacement signal. Compared with the vision-based measurement, the normalised root mean square deviation of GPS measurement was decreased from $3.17 \%$ to $2.37 \%$ after applying the data fusion. The time-dependent feature of GPS noise is also clearly identified through analysis on the data acquired from different time in the test day with the standard deviation varying from $6 \mathrm{~mm}$ to $16 \mathrm{~mm}$. 
Finally although the vision-based system is not the focus of this paper, this study is also a good demonstration about the potential of vision-based system for long-range monitoring.

\section{Acknowledgements}

The field test was possible via permission of Humber Bridge Board and was assisted by James Bassitt from University of Exeter and Mungo Morgan from Imetrum Ltd. The Humber Bridge monitoring system was installed using funding from EPSRC grant EP/F035403/1. Finally the authors would like to thank the three anonymous reviewers for their constructive comments.

\section{References}

[1] Brownjohn JMW, Koo K-Y, Scullion A, List DI. Operational deformations in long-span bridges. Struct Infrastruct Eng 2015;11:556-74. doi:10.1080/15732479.2014.951857.

[2] Wang N, O’Malley C, Ellingwood BR, Zureick A-H. Bridge Rating Using System Reliability Assessment. I: Assessment and Verification by Load Testing. J Bridg Eng 2011;16:854-62. doi:10.1061/(ASCE)BE.1943-5592.0000172.

[3] BBC News. Lorry tests as monitors installed in Forth Road Bridge first. BBC News Web Site 2015. http://www.bbc.co.uk/news/uk-scotland-edinburgh-east-fife-35151289 (accessed December 21, 2015).

[4] Psimoulis P, Stiros S. Measuring deflections of a short-span, railway bridge using a Robotic Total Station. J Bridg Eng 2013;18:182-5. doi:10.1061/(ASCE)BE.1943-5592.0000334.

[5] Stiros SC, Psimoulis P. Response of a historical short-span railway bridge to passing trains: 3D deflections and dominant frequencies derived from Robotic Total Station (RTS) measurements. Eng Struct 2012;45:362-71.

[6] Feng D, Feng M, Ozer E, Fukuda Y. A Vision-Based Sensor for Noncontact Structural Displacement Measurement. Sensors 2015;15:16557-75. doi:10.3390/s150716557. 
[7] Martins LL, Rebordão JM, Ribeiro AS. Structural observation of long-span suspension bridges for safety assessment: implementation of an optical displacement measurement system. J Phys Conf Ser 2015;588. doi:10.1088/1742-6596/588/1/012004.

[8] Martins LL, Nunes Vicente Rebordão JM, Silva Ribeiro Á. Thermal Influence on LongDistance Optical Measurement of Suspension Bridge Displacement. Int J Thermophys 2014;35:693-711. doi:10.1007/s10765-014-1607-3.

[9] Nickitopoulou A, Protopsalti K, Stiros SC. Monitoring dynamic and quasi-static deformations of large flexible engineering structures with GPS: Accuracy, limitations and promises. Eng Struct 2006;28:1471-82.

[10] Casciati F, Fuggini C. Engineering vibration monitoring by GPS: long duration records. Earthq Eng Eng Vib 2009;8:459-67.

[11] Yi TH, Li HN, Gu M. Experimental assessment of high-rate GPS receivers for deformation monitoring of bridge. Measurement 2013;46:420-32. doi:10.1016/j.measurement.2012.07.018.

[12] Moschas F, Stiros S. PLL bandwidth and noise in $100 \mathrm{~Hz}$ GPS measurements. GPS Solut 2014;19:173-85. doi:10.1007/s10291-014-0378-4.

[13] Sohn H, Farrar CR, Hemez F, Czarnecki J. A Review of Structural Health Monitoring Literature 1996 - 2001. Third World Conf. Struct. Control, Como, Italy: 2002. doi:LA-13976MS.

[14] Li X, Ge L, Ambikairajah E, Rizos C, Tamura Y, Yoshida A. Full-scale structural monitoring using an integrated GPS and accelerometer system. GPS Solut 2006;10:233-47. doi:10.1007/s10291-006-0023-y.

[15] Roberts GW, Meng X, Dodson AH. Integrating a Global Positioning System and Accelerometers to Monitor the Deflection of Bridges. J Surv Eng 2004;130:65-72. doi:10.1061/(ASCE)0733-9453(2004)130:2(65).

[16] Chan WS, Xu YL, Ding XL, Dai WJ. An integrated GPS-accelerometer data processing 
technique for structural deformation monitoring. J Geod 2006;80:705-19. doi:10.1007/s00190006-0092-2.

[17] Moschas F, Psimoulis PA, Stiros SC. GPS / RTS data fusion to overcome signal deficiencies in certain bridge dynamic monitoring projects. Smart Struct Syst 2013;12:251-69.

[18] Moschas F, Stiros S. Dynamic Deflections of a Stiff Footbridge Using 100-Hz GNSS and Accelerometer Data. J Surv Eng 2015;141. doi:10.1061/(ASCE)SU.1943-5428.0000146.

[19] Moschas F, Stiros S. Measurement of the dynamic displacements and of the modal frequencies of a short-span pedestrian bridge using GPS and an accelerometer. Eng Struct 2011;33:10-7. doi:10.1016/j.engstruct.2010.09.013.

[20] Yi TH, Li HN, Gu M. Wavelet based multi-step filtering method for bridge health monitoring using GPS and accelerometer. Smart Struct Syst 2013;11:331-48.

[21] Psimoulis P a., Stiros SC. A supervised learning computer-based algorithm to derive the amplitude of oscillations of structures using noisy GPS and Robotic Theodolites (RTS) records. Comput Struct 2012;92-93:337-48. doi:10.1016/j.compstruc.2011.10.019.

[22] Smyth A, Wu M. Multi-rate Kalman filtering for the data fusion of displacement and acceleration response measurements in dynamic system monitoring. Mech Syst Signal Process 2007;21:706-23. doi:10.1016/j.ymssp.2006.03.005.

[23] Chang CC, Xiao XH. An integrated visual-inertial technique for structural displacement and velocity measurement. Smart Struct Syst 2010;6:1025-39.

[24] Kim J, Kim K, Sohn H. Autonomous dynamic displacement estimation from data fusion of acceleration and intermittent displacement measurements. Mech Syst Signal Process 2014;42:194-205. doi:10.1016/j.ymssp.2013.09.014.

[25] Li Z, Chang CC. Adaptive Quantification of Noise Variance Using Subspace Technique. J Eng Mech 2013;139:469-78. doi:10.1061/(ASCE)EM.1943-7889.0000499. 
[26] Wahbeh AM, Caffrey JP, Masri SF. A vision-based approach for the direct measurement of displacements in vibrating systems. Smart Mater Struct 2003;12:785-94. doi:10.1088/09641726/12/5/016.

[27] Ye XW, Ni YQ, Wai TT, Wong KY, Zhang XM, Xu F. A vision-based system for dynamic displacement measurement of long-span bridges: algorithm and verification. Smart Struct Syst 2013;12:363-79. doi:10.12989/sss.2013.12.3_4.363.

[28] Martins LL, Rebordão JM, Ribeiro ÁS. Optical Metrology applied to 3D displacement measurement of long-span suspension bridge dynamics. In: A. Cunha, E. Caetano, P. Ribeiro GM, editor. Proc. 9th Int. Conf. Struct. Dyn., Porto, Portugal: 2014, p. 2135-42.

[29] Moschas F, Stiros S. Noise characteristics of high-frequency, short-duration GPS records from analysis of identical, collocated instruments. Meas J Int Meas Confed 2013;46:1488-506. doi:10.1016/j.measurement.2012.12.015.

[30] Yuen K-V, Hoi K-I, Mok K-M. Selection of noise parameters for Kalman filter. Earthq Eng Eng Vib 2007;6:49-56. doi:10.1007/s11803-007-0659-9.

[31] Time History Data Files from El Centro Site Imperial Valley Irrigation District n.d. http://www.vibrationdata.com/elcentro_NS.dat.

[32] Sims C. Matlab optimization software 1999.

[33] Ashkenazi V, Roberts GW. Experimental Monitoring of the Humber Bridge Using Gps. Proc Inst Civ Eng - Civ Eng 1997;120:177-82. doi:10.1680/icien.1997.29810.

[34] Brownjohn JMW, Botfield T. A folded pendulum isolator for evaluating accelerometer performance. Exp Tech 2009;33:33-7.

[35] Khuc T, Necati Catbas F. Completely contactless structural health monitoring of real-life structures using cameras and computer vision. Struct Control Heal Monit 2016;24.

[36] Bing P, Hui-min X, Bo-qin X, Fu-long D. Performance of sub-pixel registration algorithms in 
digital image correlation. Meas Sci Technol 2006;17:1615-21. doi:10.1088/0957$0233 / 17 / 6 / 045$

[37] Busca G, Cigada A, Mazzoleni P, Zappa E. Vibration Monitoring of Multiple Bridge Points by Means of a Unique Vision-Based Measuring System. Exp Mech 2013;54:255-71. doi:10.1007/s11340-013-9784-8.

[38] Potter KD, Setchell C. Positional measurement of a feature within an image. U.S. Patent $8,718,403,2014$

[39] Brownjohn JMW, Magalhães F, Caetano E, Cunha A. Ambient vibration re-testing and operational modal analysis of the Humber Bridge. Eng Struct 2010;32:2003-18. doi:10.1016/j.engstruct.2010.02.034.

[40] Welch PD. The Use of Fast Fourier Transform for the Estimation of Power Spectra: A Method Based on Time Averaging Over Short, Modified Periodograms. IEEE Trans Audio Electroacoust 1967;15:70-3. doi:10.1109/TAU.1967.1161901.

[41] Rahbari R, Niu J, Brownjohn JMW, Koo KY. Structural identification of Humber Bridge for performance prognosis. Smart Struct Syst 2015;15:665-82. doi:10.12989/sss.2015.15.3.665.

[42] Khuc T, Catbas FN. Computer vision-based displacement and vibration monitoring without using physical target on structures. Struct Infrastruct Eng 2016;2479:1-12. doi:10.1080/15732479.2016.1164729.

[43] Hester D, Brownjohn J, Bocian M, Xu Y. Low cost bridge load test: calculating bridge displacement from acceleration for load assessment calculations. Eng Struct 2017;143:358-74. 